\title{
Transcriptional Profiling of Rice Leaves Undergoing a Hypersensitive Response Like Reaction Induced by Xanthomonas oryzae pv. oryzae Cellulase
}

\author{
Gopaljee Jha • Hitendra Kumar Patel • \\ Madhumita Dasgupta • Ramesh Palaparthi • \\ Ramesh V. Sonti
}

Received: 14 August 2009/Accepted: 20 November 2009/Published online: 22 December 2009

(C) Springer Science+Business Media, LLC 2009

\begin{abstract}
A secreted cellulase of Xanthomonas oryzae pv. oryzae induces innate immune responses in rice including a hypersensitive response (HR) like reaction. Microarray analysis was conducted using RNA isolated $12 \mathrm{~h}$ following ClsA treatment of leaves. BLAST searches were performed for the 267 (152 up- and 115 downregulated) differentially expressed ( $\geq 2$-fold) genes. A number of defense and stressresponse functions are upregulated while a number of functions involved in metabolism and transport are downregulated following induction of HR. A significant proportion of the differentially expressed genes (41/267) are predicted to encode transcription factors. Co-infiltration of $X$. oryzae pv. oryzae suppresses ClsA-induced expression of two transcription factors, OSAP2/ethylene response factor $(E R F)$ and $O s R E R J 1$, that are predicted to be involved in the jasmonic-acid-mediated defense pathway. Transient transfer of OSAP2/ERF via Agrobacterium results in the induction of
\end{abstract}

Gopaljee Jha, Hitendra Kumar Patel, and Madhumita Dasgupta contributed equally to the paper.

Electronic supplementary material The online version of this article (doi:10.1007/s12284-009-9033-z) contains supplementary material, which is available to authorized users.

G. Jha $\cdot$ H. K. Patel $\cdot$ M. Dasgupta $\cdot$ R. Palaparthi $\cdot$

R. V. Sonti $(\square)$

Centre for Cellular and Molecular Biology,

Council of Scientific and Industrial Research,

Uppal Road, Hyderabad 500007 A.P., India

e-mail: sonti@ccmb.res.in

Present Address:

G. Jha

Institute of Himalayan Bioresource Technology,

Council of Scientific and Industrial Research,

Palampur 176061 Himachal Pradesh, India callose deposition, programmed cell death, and resistance against subsequent $X$. oryzae pv. oryzae infection.

Keywords Cell wall degrading enzymes · Innate immunity · Plant defense response - Jasmonic acid · Hypersensitive response $\cdot$ Microarray $\cdot$ Programmed cell death

\section{Introduction}

Plants have powerful innate immune responses that help them ward off most potential pathogens. These immune responses are triggered following recognition of common microbe-associated molecular pattern molecules (MAMPS) or pathogen-associated molecular pattern molecules (He et al. 2007; Bent and Mackey 2007). For bacterial pathogens, such MAMPS include elicitors such as lipopolysaccharides (Newman et al. 2002), cold shock protein (Felix and Boller 2003), flagellin (Felix et al. 1999), elongation factor $\mathrm{Tu}$ (Kunze et al. 2004), an extracellular signaling molecule called AvrXa21 (Lee et al. 2006), etc. The recognition of these signals occurs through receptors at the plant cell surface (Zipfel et al. 2004, 2006; Lee et al. 2006; He et al. 2007; Bent and Mackey 2007). Bacterial pathogens have the capacity to suppress innate immune responses of plants (Palva et al. 1993; Keshavarzi et al. 2004; Li et al. 2005; Jha et al. 2007). This ability of plant pathogenic bacteria to suppress host innate immunity is considered to be a precondition for their ability to cause disease (Grant et al. 2006; Bent and Mackey 2007; Jha et al. 2007; He et al. 2007).

The plant cell wall is a formidable barrier for potential pathogens. Cell wall degrading enzymes are important virulence factors of phytopathogenic bacteria (reviewed in Jha et al. 2005). Conversely, the oligosaccharides that are 
released following the action of these enzymes on plant cell walls elicit potent innate immune responses (Darvill and Albersheim 1984; Ryan and Farmer 1991). These immune responses are in turn suppressible by plant pathogenic bacteria (Palva et al. 1993; Jha et al. 2007). Prior treatment of tobacco with purified preparations of Erwinia carotovora subsp. carotovora pectate lyase and polygalacturonase induced resistance against subsequent infection by the same pathogen (Palva et al. 1993). These defense responses are mediated by the jasmonic acid (JA)- and ethylene (ET)dependent pathways but not the salicylic acid (SA) pathway (Norman-Setterblad et al. 2000; Vidal et al. 1998).

The rice pathogen, Xanthomonas oryzae pv. oryzae, uses a bacterial Type 2 secretion system (T2S) to secrete several cell-wall-degrading enzymes including two cellulases (ClsA and EglXoB), cellobiosidase (CbsA), lipase/esterase (LipA), and xylanase (Rajeshwari et al. 2005; Jha et al. 2007; Hu et al. 2007). The EglXoB protein is expressed only during in planta growth, but all five enzymes are important for $X$. oryzae pv. oryzae virulence. Conversely, treatment with either ClsA or CbsA or LipA induces innate immune responses such as HR like reaction and callose deposition in rice (Jha et al. 2007). These defense responses are so potent that prior treatment with these cell-walldegrading enzymes induces resistance against subsequent infection by $X$. oryzae pv. oryzae. It appears that soluble elicitors that are released by the action of these enzymes on rice walls are the actual elicitors of innate immune responses. These defense responses are suppressed by $X$. oryzae pv. oryzae using proteins that are secreted through the type 3 secretion system (T3S). Prior treatment with a $\mathrm{T}^{3} \mathrm{~S}^{-} X$. oryzae pv. oryzae mutant induces resistance against subsequent infection by the wild-type pathogen indicating that defense suppression is crucial for the ability of $X$. oryzae pv. oryzae to cause disease. A $\mathrm{T}_{2} \mathrm{~S}^{-} \mathrm{T}^{-} \mathrm{S}^{-}$ double mutant of $X$. oryzae pv. oryzae is very much compromised in the ability to elicit rice innate immune responses indicating that a functional T2S and presumably the cell-wall-degrading enzymes that are secreted through this system play an important role in elicitation of innate immunity during growth within the plant (Jha et al. 2007).

Secreted ClsA is a $48-\mathrm{kDa}$ protein that exhibits endoglucanase as well as exoglucanase activities. In order to understand the molecular events associated with ClsA-induced innate immune responses in rice, we have performed transcriptome analysis following treatment with the ClsA protein at concentrations that would induce HR. Our results indicated that a number of functions that are related to defense and stress are upregulated while those that are related to metabolism and transport are downregulated following ClsA treatment. A number of transcription factor genes are differentially expressed during cellulase-induced HR. Transient transfer of one of these transcription factor genes, OsAP2/ethylene response factor
$(E R F)$, via Agrobacterium, results in induction of callose deposition in rice leaves, programmed cell death (PCD) in rice roots, and enhanced resistance against subsequent $X$. oryzae pv. oryzae infection.

\section{Results}

Microarray analysis of ClsA-induced transcriptome of rice

In order to understand gene expression changes in rice leaves undergoing a ClsA-induced HR, we performed microarray analysis using the Affymetrix GeneChip rice genome array. Each array contains probe sets for 51,279 rice transcripts; out of which, around 48,564 are from japonica and 1,260 from indica cultivars. The adaxial surfaces of leaves of 15 days old Taichung Native-1 (TN-1) rice seedlings were infiltrated using a needleless syringe with either $X$. oryzae pv. oryzae ClsA $(500 \mu \mathrm{g} / \mathrm{ml})$ or buffer. Approximately, $10 \mu \mathrm{l}$ of the solution is infiltrated into each leaf. The zone of infiltration is approximately $1 \mathrm{~cm}^{2}$. This means that $5 \mu \mathrm{g}$ of ClsA is infiltrated into an area of approximately $1 \mathrm{~cm}^{2}$. Total RNA was isolated, $12 \mathrm{~h}$ after treatment, from leaf tissues encompassing the zone of infiltration. The samples from 20-30 infiltrated leaves were pooled together to obtain enough RNA for the analysis. Following a series of steps (as described in the "Methods"), the RNA was converted into biotin-labeled cRNA and hybridized to the rice gene chip. Separate gene chips were hybridized for the ClsA and buffertreated samples, and the experiments were repeated with three independent biological replicates. All the raw data files (CEL files; three for ClsA and three for buffer treatment) obtained from GeneChip Operating Software (GCOS; Affymetrix) were subjected to AVADISTM Software (Strand Life Science, Bangalore, India) for further analysis. The Probe Logarithmic Intensity Error (PLIER; Affymetrix Inc 2005) and Robust Multichip Average (RMA; Irizarry et al. 2003a, b) algorithms used in the analysis picked different numbers of genes at $p<0.05$. PLIER identified 1,582 genes as differentially expressed with $\geq 1.5$-fold change and 427 genes with $\geq 2.0$-fold change at $p<0.05$ whereas RMA picked 1,004 and 302 genes with 1.5- and 2.0-fold change, respectively, at $p<0.05$. In order to reduce the possibility of false discovery, we adopted a stringent criterion wherein we considered only those genes as differentially expressed, which were selected by both the algorithms. A total of 862 genes were common to both lists at 1.5 -fold change and $p<$ 0.05. $Q$ values were calculated from $p$ values of all 862 genes, and $q$-value-based significance analysis suggested that the expected false positives are only four genes out of this list. The gene ontology (GO) enrichment analysis of $\geq 1.5$ fold change rice genes differentially expressed upon ClsA treatment have revealed that genes with GO terms associated 
with response to stress are overrepresented among upregulated genes while those associated with primary and secondary metabolism, photosynthesis, chloroplast, and plastids are overrepresented among downregulated genes. Genes with GO terms associated with calcium and magnesium ion binding are also overrepresented in upregulated genes while the genes involved in iron ion binding, transporter activity, lipid metabolism, and fatty acid biosynthesis are overrepresented among downregulated genes.

All the six raw CEL files and the corresponding Avadis ${ }^{\mathrm{TM}}$ processed RMA and PLIER normalized data files have been deposited at Gene Expression Omnibus (http://www.ncbi.nlm. nih.gov/geo) under the accession number GSE8216.

\section{Rice metabolic pathway analysis}

In order to understand the changes associated with metabolic pathways during ClsA-induced HR, pathway analysis was performed using RiceCYC 1.2 Software (http://dev.gramene. org/pathway). For this analysis, we selected genes that were identified as being differentially expressed at $\geq 1.5$-fold and $p \leq 0.05$ by both PLIER and RMA algorithms. Out of the 862 genes deemed to be differentially expressed (363 upregulated and 499 downregulated), we could retrieve the requisite locus information for only 709 genes from the TIGR rice genome database. Out of these, 197 genes could be associated with different metabolic pathways using the RiceCYC 1.2. Interestingly, two paralogous genes encoding putative 12-oxo phytodienoate reductases that are predicted to be involved in JA biosynthesis as well as four different glutathione- $S$-transferases were upregulated following ClsA treatment (Supplementary Table S1). A number of plant glutathione- $S$-transferases have been reported to be upregulated following pathogen infection (Marrs 1996), and silencing of the gene for a glutathione- $S$-transferase of Nicotiana benthamiana has been shown to lead to enhanced susceptibility to Colletotrichum orbiculare (Dean et al. 2005). Five putative lipases that are predicted to be involved in triacylglycerol degradation were downregulated. Several lipase-like functions have been shown to be associated with the SA-mediated defense pathway (Falk et al. 1999; Jirage et al. 1999; Kumar and Klessig 2003).

Functional categorization of the differentially expressed genes

A majority of the differentially expressed rice genes that were identified from above were not functionally annotated in public databases. We performed BLASTX-based homology searches for 267 genes that were identified as being differentially expressed using both PLIER and RMA at $\geq 2$.0-fold change and $p<0.05$ at the NCBI server (Altschul et al. 1997) and the function of the protein with which they demonstrated significant homology $\left(E \geq 10^{-7}\right)$ was ascribed to them. We used the 2.0-fold change cutoff for this analysis as each gene was individually analyzed and the smaller number of genes ( 267 with 2.0-fold cutoff as opposed to 862 obtained with 1.5fold cutoff) was easier for us to handle. By this method, we could assign a putative function to nearly $80 \%(215 / 267)$ of the differentially expressed genes. Of these 215 genes, an $E>$ $10^{-10}$ was used to assign a function for 205 genes, and a value in the range of $10^{-7}$ to $10^{-10}$ had to be used to assign a function for about 10 genes. For the remaining genes, a function could not be assigned even if we considered less stringent $E$ values, and these genes were put in the category of "others." Based upon the assigned function, the differentially expressed genes were categorized into 14 different functional categories (Fig. 1; Tables 1 and 2). The functional categories of Bevan et al. (1998) were used with some minor changes as described in "Methods." A large number of putative transcription factors were present among the upregulated (26/152; $17 \%)$ as well as downregulated $(15 / 115 ; 13 \%)$ genes. In comparison, approximately $6 \%$ of the total genes in the rice genome are predicted to encode transcription factors (Gao et al. 2006). Interestingly, disease/defense and stress-related genes constituted $14 \%$ and $9 \%$ of the upregulated genes, respectively, whereas each of them constituted $6 \%$ of the ClsA-downregulated genes. The genes under the categories of metabolism and transport constituted $19 \%$ and $10 \%$ of the downregulated genes, respectively. These categories constituted $7 \%$ and $2 \%$ of the upregulated genes. Furthermore, genes involved in protein synthesis/turnover constituted $4 \%$ of the ClsA-upregulated genes and $\sim 1 \%$ of the downregulated genes. Several metallothioneins and stress responsive proteins like heat shock proteins as well as a peroxidase and glutathione $S$ transferase were significantly upregulated (Table 1 ). The gene for rice ortholog of Arabidopsis GUN4, a positive regulator of chlorophyll synthesis (Larkin et al. 2003) as well as several genes encoding chlorophyll $\mathrm{A} / \mathrm{B}$ binding proteins, glycine rich proteins, cytokinin dehydrogenases, and auxin responsive proteins were downregulated during ClsA-induced HR (Table 2). An important host susceptibility factor (Os8N3), whose expression is highly upregulated during $X$. oryzae pv. oryzae pathogenesis in rice (Yang et al. 2006), was $\sim 2.6$-fold downregulated during ClsA-induced HR. Also downregulated were several condensing enzymes (Os.7669.1.S1_at, Os.27713.1.A1_at, Os.9724.1.S1_at) and a B-ketoacyl reductase (Os.12839.1.S1_at; Table 2) that are predicted to function in long chain fatty acid synthesis.

Increased expression of functions involved in the JA-mediated defense pathway

during ClsA-induced HR

A rice transcription factor (OsRERJ1) gene that had been previously found to be induced by methyl JA treatment 
Fig. 1 Functional categorization of differentially expressed rice genes. The functions of differentially expressed genes ( $\geq 2$-fold change) were classified into 14 different categories based upon BLASTX analysis. The number of up- and downregulated genes for each functional category is shown in the histogram. Percentage representations of the number of genes present per individual category with respect to total up- or downregulated genes, respectively, are shown in the pie charts. The functional categories are: $a$ cell growth/division; $b$ cell structure; $c$ disease/defense; $d$ energy; $e$ intracellular traffic; $f$ metabolism; $g$ other; $h$ protein synthesis/turnover; $i$ secondary metabolism; $j$ signal transduction; $k$ stress;

$l$ transcription; $m$ transporter; $n$ transposons.

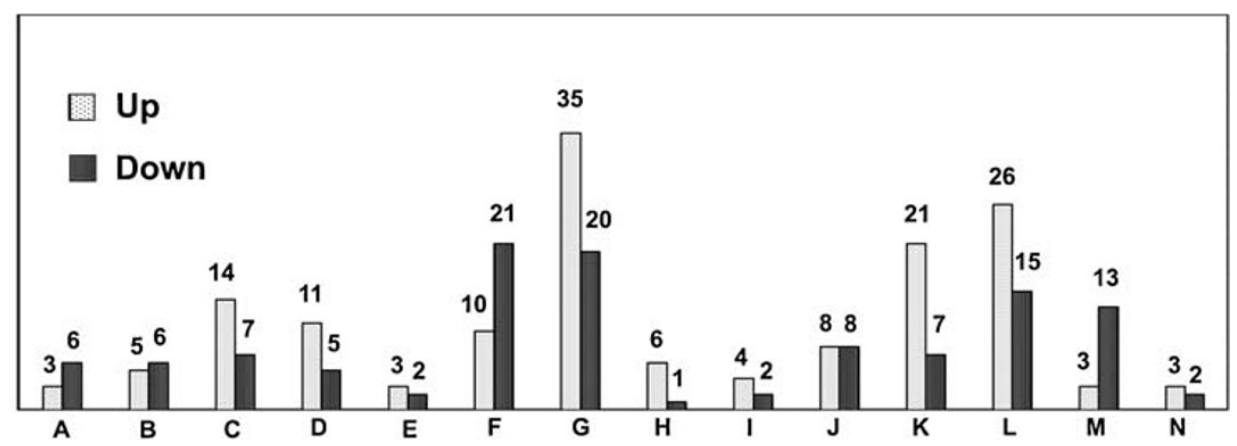

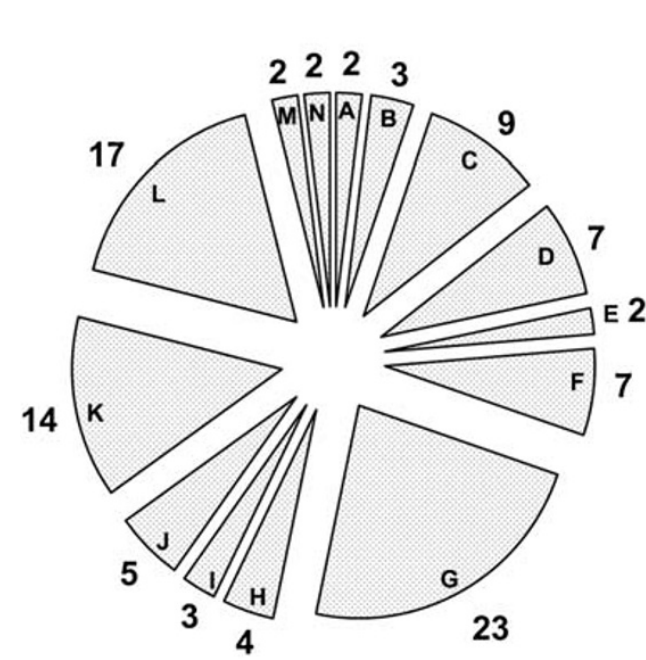

Up regulated

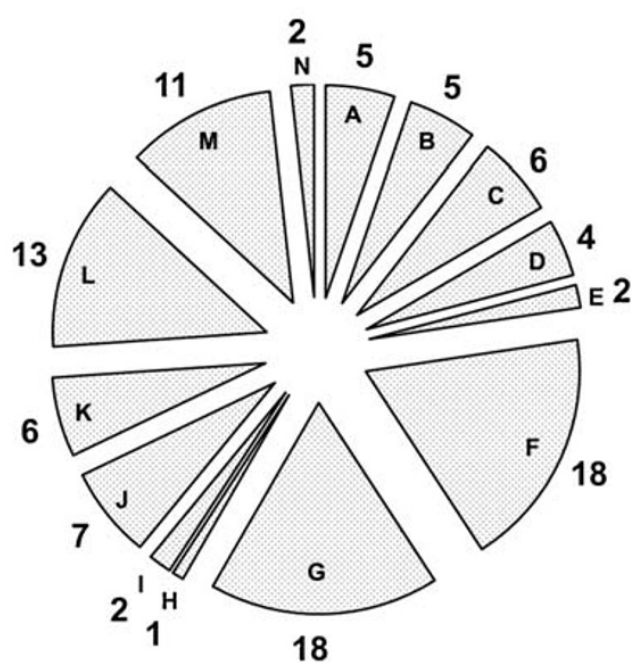

Down regulated
(Kiribuchi et al. 2004) was upregulated ( 5.3-fold) following ClsA treatment. The gene for a putative ethylene binding protein/APETALA2 type of transcription factor (OsAP2/ERF) was upregulated (4- to 7-fold) following ClsA treatment. Two different probe sets for this gene are present on this chip: one probe set (Os.15849.1.S1_s_at) exhibited a 4.3-fold upregulation while the other (OsAffx.17366.1.S1_at) exhibited a 6.5fold upregulation. This gene is homologous to subfamily 3 of AP2/ERF transcription factors in Arabidopsis; one member of this subfamily (AtERF1) is involved in integration of the JA and ET pathways (Lorenzo et al. 2003) whereas another member (AtERF2) functions as an activator in JA signaling (McGrath et al. 2005). The genes for five ZIM motif family proteins are upregulated following ClsA treatment (Table 3). The Arabidopsis homologs of four of these proteins have been shown to be upregulated by JA (Chini et al. 2007; Thines et al. 2007). These proteins function as negative regulators of the JA signaling pathway, and their upregulation has been attributed to serve in dampening the JA pathway after its initial induction. Furthermore, the ZIM family proteins repress a MYC transcription factor, which is the key activator of the JA signaling pathway (Lorenzo et al. 2004). A gene for the rice ortholog (Os.46443.1.s1_at) of this MYC factor (OsMYC) is upregulated 1.49 -fold by $\mathrm{ClsA}$ treatment.

Allene oxide synthase is a key enzyme in the JA biosynthetic pathway (Turner et al. 2002). The Affymetrix rice array contains two paralogous genes that encode allene oxide synthase. One of them (Affymetrix probe set ID, Os.8266.1.A1_at) was found to be upregulated $\sim 2.5$-fold by the PLIER algorithm and $\sim 1.95$-fold by RMA but was not identified at $p<0.05$. The other gene (Affymetrix probe set ID, Os.7678.1.S1_at) has been earlier shown to be involved in JA biosynthesis in rice (Mei et al. 2006). It was identified as being upregulated by the PLIER algorithm $(\sim 1.7$-fold at $p<$ $0.05)$ but was not selected as being upregulated using RMA. We assessed the expression of this gene (Os.7678.1.S1_at) by quantitative real-time PCR and found that it was upregulated $\sim 2.0$-fold at $p<0.05$ following ClsA treatment (Fig. 3; Supplementary Table S2, for the list of primers). Furthermore, as described above, pathway analysis had revealed the upregulation of two 12-oxophytodienoate reductase genes (OsOPR2, Os.50513.1.S1_at; OsOPR1, Os.8778.1.S1_a_at) that are predicted to be involved in JA biosynthesis (Table 3). ClsA treatment also induced the expression of two paralogous 
Table 1 Rice Genes Upregulated ( $\geq 2$ Fold) Following Cellulase Treatment

\begin{tabular}{|c|c|c|c|c|}
\hline Probe set ID $^{\mathrm{a}}$ & Public ID ${ }^{\mathrm{b}}$ & $\mathrm{FCA}^{\mathrm{c}}$ & Gene description $^{\mathrm{d}}$ & Homolog $(\text { E score })^{\mathrm{e}}$ \\
\hline \multicolumn{5}{|l|}{ Cell growth/division } \\
\hline Os.10388.1.S1_at & AK120374 & 2.74 & MEE59 (maternal effect embryo arrest 59) & NP_195447 (3e-22) \\
\hline OsAffx.7876.1.S1_s_at & $9640 . \mathrm{m} 04245$ & 2.46 & $\begin{array}{l}\text { Os12g0626500 (Seed maturation protein } \\
\text { domain containing protein) }\end{array}$ & NP_001067326 (2e-35) \\
\hline Os.38249.2.S1_at & CB638262 & 2.39 & Putative early flowering 3 & BAA83571 (6e-28) \\
\hline \multicolumn{5}{|l|}{ Cell structure } \\
\hline OsAffx.22303.1.S1_at & $9635 . \mathrm{m} 03076$ & 5.06 & $\begin{array}{l}\text { Expansin-like B1 precursor (OsEXLB1) } \\
\text { (Expansin-related 1) }\end{array}$ & Q850K7 (3e-147) \\
\hline Os.37723.1.S1_at & AK072531 & 2.27 & Putative tonoplast membrane integral protein & AAS98488 (3e-95) \\
\hline Os.5754.1.S1_at & AK099590 & 2.23 & Putative plastid developmental DAG protein & BAD03018 (8e-91) \\
\hline Os.36975.3.S1_x_at & NM_189551 & 2.17 & Putative MAR binding filament-like protein 1 & BAD68082 (0.0) \\
\hline Os.24307.1.S1_at & AK066849 & 2.01 & $\begin{array}{l}\text { TMP14 (THYLAKOID MEMBRANE } \\
\text { PHOSPHOPROTEIN OF } 14 \text { KDA) }\end{array}$ & NP_566086 (1e-24) \\
\hline \multicolumn{5}{|l|}{ Disease/defense } \\
\hline Os.53444.1.S1_at & AK072661 & 4.61 & Putative pathogen-induced protein $2-4$ & BAD08038 (7e-17) \\
\hline Os.11575.3.S1_x_at & AK065363 & 3.04 & Ankyrin-repeat protein/OsNPR3 & $\operatorname{ABE} 11617(0.0)$ \\
\hline Os.10546.1.S1_s_at & AU183565 & 2.92 & $\begin{array}{l}\text { Putative UDP-glucose:salicylic acid } \\
\text { glucosyltransferase }\end{array}$ & BAD34356 (2e-33) \\
\hline OsAffx.30138.1.S1_at & 9637.m02954 & 2.9 & $\begin{array}{l}\text { Putative UDP-glucose:salicylic acid } \\
\text { glucosyltransferase }\end{array}$ & BAD34356 (0.0) \\
\hline Os.8760.1.S1_a_at & AK099869 & 2.6 & Putative nodulin-like protein & BAD34227 (0.0) \\
\hline Os.36969.1.S1_at & AK120328 & 2.43 & NB-ARC domain containing protein & EAY99786 (3e-25) \\
\hline Os.11179.1.S1_at & CB647801 & 2.37 & $\begin{array}{l}\text { Ankyrin repeat family protein, putative, } \\
\text { expressed }\end{array}$ & ABF95250 (1e-43) \\
\hline Os.25266.1.S1_at & AK058891 & 2.34 & Putative beta 1,3 -glucanase & BAB63855 (2e-167) \\
\hline OsAffx.23277.2.S1_at & 9629.m01656 & 2.3 & Ribosome inactivating protein, expressed & ABF98182 (1e-08) \\
\hline Os.38249.1.S1_at & AK065173 & 2.23 & Nematode responsive protein-like & BAD45081 (0.0) \\
\hline Os.4327.1.S1_at & AF384030 & 2.21 & Seven transmembrane protein $\mathrm{MLO} 2$ & AF384030_1 (0.0) \\
\hline Os.11831.1.S1_at & AK062130 & 2.12 & $\begin{array}{l}\text { Putative benzothiadiazole-induced } \\
\text { S-adenosyl-L-methionine:salicylic acid } \\
\text { Carboxyl methyltransferasel }\end{array}$ & BAD45797 (0.0) \\
\hline Os.11575.1.S1_at & AK065952 & 2.09 & Ankyrin-repeat protein/OsNPR3 & $\mathrm{ABE} 11617(0.0)$ \\
\hline Os.15355.1.S1_x_at & AK067000 & 2.07 & Putative bacterial-induced peroxidase precursor & BAD28871 (2e-166) \\
\hline \multicolumn{5}{|l|}{ Energy } \\
\hline OsAffx.12379.1.S1_at & $9630 . \mathrm{m} 03520$ & 3.26 & $\begin{array}{l}\text { Putative oxygen-evolving enhancer protein } 3-2 \text {, } \\
\text { chloroplast precursor (OEE3) }\end{array}$ & BAD29563 (8e-61) \\
\hline OsAffx.32230.1.A1_at & X15901 & 2.96 & ATP synthase CF1 epsilon subunit & NP_039389 (4e-65) \\
\hline Os.52588.1.S1_at & AK068191 & 2.85 & $\begin{array}{l}2 \mathrm{Fe}-2 \mathrm{~S} \text { iron-sulfur cluster } \\
\text { protein-like/ferredoxin-related }\end{array}$ & BAC80058 (5e-86) \\
\hline Os.19061.1.S1_s_at & AK099499 & 2.77 & $\begin{array}{l}\text { 4Fe-4S ferredoxin, iron-sulfur binding; } \\
\text { Heat shock protein DnaJ }\end{array}$ & ABP02426 (2e-21) \\
\hline Os.26744.1.A1_at & CA762807 & 2.66 & Chloroplast photosystem I assembly protein Ycf3 & BAD81968 (7e-16) \\
\hline Os.44475.1.S1_x_at & AK121213 & 2.66 & $\begin{array}{l}\text { Putative NADH dehydrogenase } \\
\text { (ubiquinone oxidoreductase) }\end{array}$ & BAC15811 (0.0) \\
\hline Os.29056.1.S2_at & AK110782 & 2.54 & Apocytochrome f precursor, putative & AAP53263.2 (4e-54) \\
\hline Os.27830.1.S1_at & AK068377 & 2.54 & $\begin{array}{l}\text { PsbQ domain protein family/oxygen } \\
\text { evolving enhancer } 3\end{array}$ & BAC10375 (4e-105) \\
\hline OsAffx.32225.1.A1_at & $\mathrm{X} 15901$ & 2.5 & Photosystem I assembly protein Ycf3 & NP_039384 (3e-95) \\
\hline Os.3406.1.S1_at & AB004865 & 2.48 & Alternative oxidase & BAA28771 $(0.0)$ \\
\hline Os.11570.1.S1_s_at & AK101484 & 2.22 & Putative glycerol-3-phosphate dehydrogenase & AAU44049 (0.0) \\
\hline \multicolumn{5}{|l|}{ Intracellular traffic } \\
\hline Os.8700.1.S1_at & AK066218 & 2.74 & Dynein light chain type 1-like & BAD28635 (2e-36) \\
\hline Os.31464.1.S1_a_at & AK101489 & 2.6 & Os04g0527400 & NP_001053367 (0.0) \\
\hline Os.40002.1.S1_s_at & CF315096 & 2.17 & Got1-like family & NP_001060166 (1e-07) \\
\hline \multicolumn{5}{|l|}{ Metabolism } \\
\hline Os.18490.1.S1_x_at & AK120238 & 3.16 & $\begin{array}{l}\text { Thiamine biosynthesis protein ThiC, } \\
\text { putative, expressed }\end{array}$ & ABF98201 (0.0) \\
\hline Os.56682.1.S1_at & AK110292 & 3.1 & GDSL-lipase-like & BAD61697 (0.0) \\
\hline Os.23154.2.S1_at & AK111360 & 2.68 & Putative proline-rich protein APG & BAD15755 (1e-61) \\
\hline
\end{tabular}


Table 1 (continued)

\begin{tabular}{|c|c|c|c|c|}
\hline Probe set $\mathrm{ID}^{\mathrm{a}}$ & Public ID $^{\mathrm{b}}$ & $\mathrm{FCA}^{\mathrm{c}}$ & Gene description $^{\mathrm{d}}$ & Homolog $(\text { E score })^{\mathrm{e}}$ \\
\hline Os.52414.1.S1_at & AK066972 & 2.41 & $\begin{array}{l}\text { (GDSL-lipase-like) } \\
\text { Hydrolase, alpha/beta fold family } \\
\text { protein-like }\end{array}$ & BAD23358 (8e-145) \\
\hline OsAffx.27752.1.S1_s_at & 9634.m02096 & 2.41 & Putative aldose 1 -epimerase & BAD05401 (4e-149) \\
\hline Os.50951.1.S1_at & AK059666 & 2.21 & Metallo-beta-lactamase protein-like & BAD28843 (6e-136) \\
\hline OsAffx.32255.1.A1_at & X15901 & 2.19 & Acetyl-coa carboxylase beta subunit & NP_039394 (5e-56) \\
\hline Os.8957.1.S1_at & AK071523 & 2.19 & $\begin{array}{l}\text { Phosphatidic acid phosphatase-related/ } \\
\text { PAP2-related }\end{array}$ & NP_190970 (6e-128) \\
\hline Os.47381.2.S1_at & AK120916 & 2.12 & $\begin{array}{l}\text { Putative 3-oxoacyl-[acyl-carrier-protein] } \\
\text { synthase I, chloroplast precursor }\end{array}$ & BAD35225 (0.0) \\
\hline Os.8957.1.S1_a_at & AK071523 & 2.06 & $\begin{array}{l}\text { Phosphatidic acid phosphatase- } \\
\text { related/PAP2-related }\end{array}$ & NP_190970 (3e-128) \\
\hline \multicolumn{5}{|c|}{ 16 } \\
\hline OsAffx.5746.1.S1_s_at & 9636.m00746 & 8.72 & Hypothetical protein OsJ_025189 & EAZ41706 (3e-20) \\
\hline Os.4618.1.S1_at & AK062310 & 4.71 & Hypothetical protein OsI_002101 & EAY74254 (2e-50) \\
\hline Os.40007.1.S1_x_at & CF318727 & 3.86 & Os03g0120600 & NP_001048790 (4e-18) \\
\hline Os.6288.1.S1_at & AK106356 & 3.58 & Unknown protein/Os08g0412700 & NP_001061796 (2e-169) \\
\hline Os.20548.1.S1_at & AK066753 & 3.58 & Hypothetical protein OsI_016689 & EAY95456 (9e-34) \\
\hline Os.30528.1.S1_at & AK108716 & 3.45 & Unknown protein/Os08g0412800 & NP_001061797 (0.0) \\
\hline Os.46544.1.A1_at & AK066066 & 3.38 & Expressed protein/Os10g0389500 & NP_001064501 (3e-113) \\
\hline Os.21260.1.S1_at & AK067400 & 3.38 & Os03g0184100 & NP_001049187 (2e-120) \\
\hline Os.6516.1.S1_at & CF303902 & 3.37 & No significant similarity found & \\
\hline Os.52004.1.S1_at & AK064520 & 3.31 & Hypothetical protein LOC_Os12g17920 & ABA97088 (3e-06) \\
\hline Os.27218.1.A1_at & CB649864 & 3.23 & Os05g0575000 & NP_001056395 (5e-33) \\
\hline Os.57343.1.S1_at & AK111335 & 3.06 & Os02g0733900 & NP_001048037 (2e-36) \\
\hline Os.22703.1.S1_at & AK066033 & 3.03 & Unknown protein /Os07g0618700 & BAC79871 (1e-112) \\
\hline Os.27764.1.S1_at & CB629248 & 3 & Os05g0464200 & NP_001055778 (4e-04) \\
\hline Os.51529.1.S1_at & AK063010 & 2.99 & Os12g0174200 & NP_001066286 (1e-16) \\
\hline Os.39942.2.S1_x_at & CF321166 & 2.81 & Osjnbb0058j09.9 & CAD39870 (9e-06) \\
\hline Os.7944.1.S1_at & AK 120788 & 2.76 & No significant similarity found & \\
\hline Os.5823.1.S1_at & AK109216 & 2.75 & Os02g0718200 & NP_001047938 (0.003) \\
\hline Os.51299.1.S1_at & AK062470 & 2.75 & Os05g0464300 & NP_001055779 (2e-14) \\
\hline Os.4773.1.S1_at & CF325704 & 2.67 & Os06g0133500 & NP_001056709 (3e-30) \\
\hline Os.57191.1.S1_at & AK111114 & 2.63 & Os06g0147300 & NP_001056802 (2e-50) \\
\hline Os.18712.1.S1_at & AK108985 & 2.63 & Hypothetical protein OsI_028900 & EAZ07668 (7e-127) \\
\hline Os.2426.1.A1_at & CA759372 & 2.49 & Expressed protein & ABA92028 (2e-21) \\
\hline Os.12342.1.S2_at & CB668460 & 2.43 & No significant similarity found & \\
\hline Os.39725.1.S1_at & CA763989 & 2.35 & No significant similarity found & \\
\hline Os.7130.2.S1_x_at & AU032629 & 2.27 & No significant similarity found & \\
\hline OsAffx.7530.1.S1_s_at & $9640 . \mathrm{m} 00831$ & 2.26 & Expressed protein & ABA96042 (6e-24) \\
\hline Os.38992.1.A1_x_at & D43281 & 2.23 & No significant similarity found & \\
\hline OsAffx.27741.1.S1_s_at & 9634.m02034 & 2.23 & Hypothetical protein & BAD62179 (2e-21) \\
\hline Os.50952.1.S1_at & AK059668 & 2.19 & No Significant similarity & \\
\hline Os.10823.2.S1_at & AK064162 & 2.07 & Os05g0487300/unknown protein & NP_001055881 (1e-05) \\
\hline Os.55407.1.S1_at & AK108023 & 2.02 & Os03g0799300 & NP_001051576 (3e-26) \\
\hline \multicolumn{5}{|l|}{ Protein synthesis/Turnover } \\
\hline Os.55628.1.S1_at & AK 108443 & 3.69 & $\begin{array}{l}\text { Eukaryotic translation initiation factor } \\
\text { SUI1, putative }\end{array}$ & NP_175831 (3e-30) \\
\hline OsAffx.26671.1.S1_x_at & $9632 . \mathrm{m} 05851$ & 3.34 & $\begin{array}{l}\text { Eukaryotic translation initiation factor } \\
\text { SUI1, putative }\end{array}$ & $\mathrm{NP}_{-} 175831(4 \mathrm{e}-30)$ \\
\hline Os.8724.1.S1_at & AK072982 & 3.02 & Cyclin-like-F-box family protein & NP_567629 (5e-09) \\
\hline OsAffx.24862.1.S1_at & $9630 . \mathrm{m} 05377$ & 2.43 & F-box domain containing protein & AAP54337.2 (1e-27) \\
\hline Os.46325.1.A1_at & AK 120658 & 2.32 & $\begin{array}{l}\text { Zinc finger (C3HC4-type RING finger) } \\
\text { family protein }\end{array}$ & NP_568096 (3e-146) \\
\hline Os.27569.1.S2_at & AK121706 & 2.26 & Calcium-binding EF-hand family protein-like & BAD08916 (8e-49) \\
\hline
\end{tabular}

Secondary metabolism 
Table 1 (continued)

\begin{tabular}{|c|c|c|c|c|}
\hline Probe set ID $^{\mathrm{a}}$ & Public ID ${ }^{\mathrm{b}}$ & $\mathrm{FCA}^{\mathrm{c}}$ & Gene description $^{\mathrm{d}}$ & Homolog $(\text { E score })^{\mathrm{e}}$ \\
\hline Os.50053.1.A1_at & AK119780 & 3.9 & 9-cis-epoxycarotenoid dioxygenase 4 & AAW21320 (0.0) \\
\hline Os.6376.1.S1_at & AK121461 & 3.41 & Chalcone-flavanone isomerase & NP_001057086 (0.0) \\
\hline Os.27507.1.S1_at & AK109673 & 2.97 & $\begin{array}{l}\text { Reticuline oxidase precursor, putative/berberine } \\
\text { bridge enzyme-like protein }\end{array}$ & ABA93766 (1e-132) \\
\hline OsAffx.32219.1.A1_x_at & X15901 & 2.61 & $\begin{array}{l}\text { Terpene synthase family, metal binding domain } \\
\text { containing protein }\end{array}$ & ABF96083 (0.0) \\
\hline \multicolumn{5}{|l|}{ Signal transduction } \\
\hline Os.1606.1.S1_at & AK111969 & 5.05 & Putative receptor-like protein kinase & AAL87185 (8e-137) \\
\hline Os.15247.1.S1_s_at & AB125310 & 3.15 & Serine/threonine-protein kinase SAPK9 & BAD18005 $(0.0)$ \\
\hline Os.10254.1.S1_at & AK066150 & 3.12 & Copine I-like & BAD10026 (0.0) \\
\hline Os.9585.1.S1_at & AB060552 & 3.04 & Calmodulin-2 & BAB69673 (8e-66) \\
\hline Os.26226.1.S1_at & AK103704 & 2.96 & Putative serine/threonine protein kinase & BAD08131 (0.0) \\
\hline Os.9585.1.S1_s_at & AB060552 & 2.62 & Calmodulin, putative, expressed & ABA99816 (5e-66) \\
\hline Os.35013.1.S1_at & AK069274 & 2.16 & Putative Serine/threonine phosphatases & AAP06902 (0.0) \\
\hline OsAffx.11760.1.S1_at & $9629 . \mathrm{m} 06614$ & 2 & Putative S-receptor kinase & BAD82381 (0.0) \\
\hline \multicolumn{5}{|l|}{ Stress } \\
\hline Os.51546.1.S1_at & AK063042 & 10.2 & Senescence-associated protein-related & NP_197570 (1e-08) \\
\hline OsAffx.32080.1.S1_at & $9640 . \mathrm{m} 03722$ & 7.34 & $\begin{array}{l}\text { Metallothionein-like protein 1, putative, } \\
\text { expressed }\end{array}$ & ABA99660 (8e-30) \\
\hline Os.37783.1.S1_a_at & AK105219 & 4.52 & Metallothionein-like protein 4C (OsMT-I-4c) & Q2QNC3 (2e-22) \\
\hline OsAffx.7826.2.S1_at & $9640 . \mathrm{m} 03694$ & 4.02 & $\begin{array}{l}\text { Metallothionein-like protein 1, putative, } \\
\text { expressed }\end{array}$ & ABA99599 (1e-10) \\
\hline Os.37773.1.S1_at & AU165294 & 3.82 & Low molecular mass heat shock protein & AAC78392 (2e-37) \\
\hline Os.37783.2.S1_x_at & AU070898 & 3.73 & $\begin{array}{l}\text { Metallothionein-like protein 1, putative, } \\
\text { expressed }\end{array}$ & ABA99639 (4e-24) \\
\hline Os.14105.1.S1_at & AK106404 & 3.63 & Cytochrome P450 & AAX92767 (0.0) \\
\hline Os.4671.1.S1_a_at & AF435970 & 3.47 & $\begin{array}{l}\text { Jacalin-related lectin like /Protein } \\
\text { mannose-binding lectin }\end{array}$ & P24120 (2e-78) \\
\hline Os.37773.1.S1_x_at & AU165294 & 3.46 & $\begin{array}{l}\text { Alpha-crystallin-Hsps } / 17.4 \text { kda class I } \\
\text { heat shock protein, putative }\end{array}$ & AAP06883 (6e-38) \\
\hline Os.6092.1.S1_at & AB120515 & 3.17 & Trehalose-6-phosphate phosphatase & BAD12596 (0.0) \\
\hline Os.7756.1.S1_at & AK060639 & 2.7 & $\begin{array}{l}\text { Putative stress-responsive protein } \\
\text { (pectinesterase inhibitor domain } \\
\text { containing) }\end{array}$ & AAM94917 (2e-83) \\
\hline Os.15428.1.S1_at & AK065414 & 2.61 & Putative wound inducive gene & BAD81783 (2e-174) \\
\hline Os.8741.1.S1_at & AK102303 & 2.57 & RADICAL-INDUCED CELL DEATH1 & ABF94777 (0.0) \\
\hline Os.33675.1.S1_at & AK099569 & 2.5 & $\begin{array}{l}\text { Rbcx protein having a possible } \\
\text { chaperonin-like function }\end{array}$ & NP_001061837 (2e-82) \\
\hline Os.8774.1.S1_at & AK071331 & 2.43 & Peroxidase-like protein & BAD82471 (7e-127) \\
\hline Os.5363.1.S1_at & AU174652 & 2.4 & Putative cadmium-induced protein & BAC19956 (1e-29) \\
\hline Os.53236.1.S1_at & AK071507 & 2.28 & $\begin{array}{l}\text { Putative zinc finger protein/Salt } \\
\text { tolerance-like protein }\end{array}$ & BAD25819 (7e-95) \\
\hline Os.49030.1.A1_s_at & CR292984 & 2.27 & Putative glutathione S-transferase OsGSTU5 & AF309377_1 (3e-09) \\
\hline \multicolumn{5}{|l|}{ Transcription } \\
\hline OsAffx.17366.1.S1_at & $9636 . \mathrm{m} 03709$ & 6.54 & $\begin{array}{l}\text { Ethylene-binding protein-AP2 domain } \\
\text { containing }\end{array}$ & BAD38371 (4e-41) \\
\hline Os.6043.1.S1_at & AB040744 & 5.3 & $\begin{array}{l}\text { RERJ1(Helix-loop-helix domain containing } \\
\text { transcriptin factor) }\end{array}$ & AB040744 (8e-111) \\
\hline OsAffx.27442.1.S1_at & NM_185471 & 5.23 & Transcription factor CBF1 & BAA90812 (3e-73) \\
\hline Os.4463.1.S1_s_at & AY327040 & 4.57 & Transcription factor CBF1 & BAA90812 (3e-73) \\
\hline Os.15849.1.S1_s_at & AK062882 & 4.34 & $\begin{array}{l}\text { AP2 domain-containing transcription } \\
\text { factor-like }\end{array}$ & BAD17116 (4e-15) \\
\hline Os.49746.1.S1_at & AY581256 & 3.87 & $\begin{array}{l}\text { Induced protein mgi1(MYB-like } \\
\text { DNA-binding domain) }\end{array}$ & AAS90600 (1e-76) \\
\hline Os.55259.1.S1_at & AK107750 & 3.77 & ZIM motif family protein, expressed & ABF94311 (1e-07) \\
\hline Os.46849.1.S1_at & AK107854 & 3.46 & ZIM motif family protein, expressed & AAP53563 (7e-23) \\
\hline Os.54501.1.S1_at & AK105440 & 3.44 & PHD-finger, putative & AAX94898 (0.0) \\
\hline Os.5335.1.S1_at & AK101209 & 3.19 & Putative MYB transcription factor & BAD61826 (1e-33) \\
\hline Os.3400.1.S1_s_at & AB001888 & 3.05 & Zinc finger protein & BAA33206 (0.0) \\
\hline
\end{tabular}


Table 1 (continued)

\begin{tabular}{|c|c|c|c|c|}
\hline Probe set $\mathrm{ID}^{\mathrm{a}}$ & Public ID ${ }^{\mathrm{b}}$ & $\mathrm{FCA}^{\mathrm{c}}$ & Gene description $^{\mathrm{d}}$ & Homolog $(\text { E score })^{\mathrm{e}}$ \\
\hline Os.12977.1.S1_at & AK072192 & 2.96 & $\begin{array}{l}\text { Zinc finger protein/COL9 } \\
\text { (CONSTANS-LIKE 9); } \\
\text { transcription factor }\end{array}$ & BAA33206 (4e-141) \\
\hline Os.55550.1.S1_at & AK108297 & 2.88 & Transcription factor GT-3b & AAP13348 (3e-04) \\
\hline Os.9923.1.S1_s_at & AK120087 & 2.58 & ZIM motif family protein, expressed & ABF94311 (9e-57) \\
\hline Os.35681.1.S1_at & AY344493 & 2.54 & Putative heat shock transcription factor 8 & BAB68070 (2e-124) \\
\hline Os.35343.1.A1_at & AK067922 & 2.51 & $\begin{array}{l}\text { No apical meristem protein (NAC domain } \\
\text { containing) }\end{array}$ & ABA95706 (2e-116) \\
\hline Os.10411.1.S1_at & AY077725 & 2.45 & $\mathrm{C} 2 \mathrm{H} 2$ zinc finger protein & BAD81699 (2e-72) \\
\hline Os.23778.1.S1_at & AK101934 & 2.44 & Putative heat stress transcription factor & BAD25410 (0.0) \\
\hline Os.7130.1.S1_at & AK073475 & 2.42 & $\begin{array}{l}\text { Transcription factor jumonji, putative, } \\
\text { expressed }\end{array}$ & ABB48025 (0.0) \\
\hline Os.8031.1.S1_at & AK111775 & 2.38 & $\begin{array}{l}\text { Putative ethylene response factor } 2 / \mathrm{AP} 2 \\
\text { domain-containing transcription factor }\end{array}$ & BAB92777 (1e-73) \\
\hline Os.49787.1.S1_at & AK111571 & 2.36 & Putative MYB-like transcription factor & AAT69605 (6e-50) \\
\hline Os.20750.1.S1_at & CB635910 & 2.33 & bZIP transcription factor-like & BAB89012 (5e-54) \\
\hline Os.19899.1.S1_x_at & AK106830 & 2.12 & Transcription factor PCF8 & BAC01119 (4e-38) \\
\hline Os.11046.1.S1_at & AK119729 & 2.1 & $\begin{array}{l}\text { Putative ethylene-responsive transcriptional } \\
\text { coactivator }\end{array}$ & BAD32863 (8e-60) \\
\hline Os.10356.1.S1_at & AK073589 & 2.09 & ZIM motif family protein, expressed & ABF94310 (2e-82) \\
\hline OsAffx.3006.1.S1_at & $9630 . \mathrm{m} 04817$ & 2.08 & MYB-like transcription factor-like & BAD13038 (1e-15) \\
\hline \multicolumn{5}{|l|}{ Transporter } \\
\hline Os.47946.1.S1_s_at & AK070124 & 4.22 & $\begin{array}{l}\text { Putative glucose-6-phosphate/phosphate- } \\
\text { translocator precursor }\end{array}$ & BAC57673 (2e-90) \\
\hline Os.11800.1.S1_s_at & AK108373 & 3.37 & MDR-like ABC transporter & CAD59587 (3e-141) \\
\hline Os.11800.1.S1_at & AK108373 & 3.13 & MDR-like ABC transporter & CAD59587 (4e-140) \\
\hline \multicolumn{5}{|l|}{ Transposons } \\
\hline Os.18078.1.S1_at & C97547 & 3.91 & Rtac1 & AAX95969 (0.17) \\
\hline Os.39994.1.S1_at & CF319063 & 3.8 & Rtac1 & AAL86017 (1e-10) \\
\hline Os.6901.1.S1_at & AK102892 & 2.72 & $\begin{array}{l}\text { Os } 10 \mathrm{~g} 0378500 / \text { retrotransposon protein, } \\
\text { putative }\end{array}$ & NP_001064482 (2e-31) \\
\hline
\end{tabular}

${ }^{a}$ Affymetrix Probe set ID of upregulated gene

${ }^{\mathrm{b}}$ The public representative ID of these genes obtained from Affymetrix NetAffx analysis center

${ }^{\mathrm{c}}$ PLIER normalized absolute fold change value for upregulation

${ }^{d}$ The putative function of the gene based on BLASTX analysis

${ }^{\mathrm{e}}$ Accession ID of the homologous protein based upon which the function has been assigned. The corresponding BLASTX E score is shown in brackets

${ }^{\mathrm{f}}$ Genes encoding hypothetical proteins or those with no significant matches in the databases are kept in this category

rice chitinase $(P R-3)$ genes whose Arabidopsis ortholog is involved in a JA-mediated defense pathway (Thomma et al. 1998). ClsA treatment led to increased expression of a rice ortholog of the Arabidopsis RADICAL-INDUCED CELL $D E A T H-1$ gene which has been postulated to modulate methyl JA responses (Ahlfors et al. 2004). A gene encoding a jacalin-related lectin like protein which is homologous to a JA-induced protein of wheat and barley (Lee et al. 1996; Wang and Ma 2005) was $~ 3.5$-fold induced by ClsA treatment.

The gene for the rice ortholog of Arabidopsis COPINE1 (a negative regulator of SA-associated systemic-acquired resistance; Jambunathan et al. 2001) was $\sim 3$-fold upregulated during ClsA-induced HR. Interestingly, genes that are putatively involved in SA modification were upregulated.
Two different probe sets (Os.10546.1.S1_s_at and OsAffx.30138.1.S1_at) for a gene encoding a putative UDPglucose/salicylic acid glucosyltransferase (OSSGT; predicted to be involved in glucosylation and inactivation of SA; Chen et al. 1995) were upregulated $\sim 2.9$-fold. The gene for a putative benzothiadiazole-induced $S$-adenosyl-L-methionine: salicylic acid carboxyl methyltransferase1 (OSBSMT1; which is predicted to be involved in volatilizing SA; Koo et al. 2007) was 2 .0-fold upregulated. Glycosylated SA is an inactive storage form of SA (Chen et al. 1995) whereas methylated SA is volatile (Koo et al. 2007); thus, both modifications might lead to overall reduction of the cellular pool of SA. Increased expression of the JA pathway has been suggested to lead to a downregulation of the SA pathway (Takahashi et al. 2004; Kunkel and Brooks 2002; Spoel et al. 2003). 
Table 2 Rice Genes Downregulated ( $\geq 2$-Fold) Following Cellulase Treatment

\begin{tabular}{|c|c|c|c|c|}
\hline Probe set $\mathrm{ID}^{\mathrm{a}}$ & Public ID ${ }^{\mathrm{b}}$ & $\mathrm{FCA}^{\mathrm{c}}$ & Gene description $^{\mathrm{d}}$ & Homolog $(\text { E score })^{\mathrm{e}}$ \\
\hline \multicolumn{5}{|l|}{ Cell growth/division } \\
\hline Os.27224.2.A1_at & AK103510 & 3.66 & Putative cytokinin dehydrogenase & BAD09964 (8e-59) \\
\hline OsAffx.29543.1.S1_x_at & $9636 . \mathrm{m} 03606$ & 3.05 & Putative cytokinin dehydrogenase & BAD09964 (0.0) \\
\hline Os.43596.1.S1_at & CB620528 & 2.62 & Cytokinin oxidase/dehydrogenase & BAE16612 (3e-39) \\
\hline Os.9392.1.S1_at & AK071762 & 2.16 & Auxin/aluminum-responsive protein putative & NP_197415 (3e-86) \\
\hline Os.55455.1.S1_at & AK108118 & 2.06 & Auxin-responsive protein-like & BAD25785 (2e-26) \\
\hline Os.12201.1.S1_at & AK065039 & 2.05 & $\begin{array}{l}\text { Putative 1-aminocyclopropane-1-carboxylate } \\
\text { oxidase } 1 \text { (ACC oxidase } 1)\end{array}$ & BAD38208 (1e-171) \\
\hline \multicolumn{5}{|l|}{ Cell structure } \\
\hline Os.49281.1.S1_s_at & AK062457 & 4.40 & Putative glycine rich protein & BAD61563 (1e-11) \\
\hline Os.38152.1.S1_at & AK065615 & 3.41 & LrgB-like family protein expressed & AAP55171 (1e-73) \\
\hline OsAffx.11838.1.S1_at & $9629 . \mathrm{m} 07254$ & 2.53 & Putative bundle sheath cell specific protein 1 & BAB63632 (8e-60) \\
\hline Os.55444.1.S1_at & AK108103 & 2.34 & Glycine-rich protein & NP_193893 (4e-08) \\
\hline Os.10370.1.S1_at & AK062370 & 2.32 & Putative glycine rich protein & BAD61558 (1e-14) \\
\hline Os.10370.1.S1_x_at & AK062370 & 2.27 & Putative glycine rich protein & BAD61558 (1e-14) \\
\hline \multicolumn{5}{|l|}{ Disease/defense } \\
\hline Os.10401.1.S1_s_at & AK070510 & 2.65 & Putative MtN3 (disease resistant allele XA13) & BAD13168 (1e-141) \\
\hline Os.51641.1.S1_at & AK063248 & 2.55 & Putative pathogenesis-related protein/PR1 like & BAC84818 (2e-67) \\
\hline Os.37893.1.S1_at & AK100346 & 2.46 & Phenylalanine ammonia-lyase & AAO72666 (0.0) \\
\hline Os.37890.1.S1_s_at & AK061288 & 2.09 & $\begin{array}{l}\text { Protease inhibitor/seed storage/Lipid Transfer } \\
\text { Protein family putative }\end{array}$ & AAX96637 (4e-39) \\
\hline Os.9679.1.S1_at & AK060077 & 2.03 & $\begin{array}{l}\text { Disease resistance-responsive family protein } \\
\text { putative expressed }\end{array}$ & ABA91787 (1e-90) \\
\hline Os.46305.1.S1_at & AK122171 & 2.01 & Leucine Rich Repeat family protein expressed & ABA96188 (0.0) \\
\hline Os.51641.1.S1_x_at & AK063248 & 2.86 & Putative pathogenesis-related protein/ PR1 like & BAC84818 (5e-68) \\
\hline \multicolumn{5}{|l|}{ Energy } \\
\hline Os.12181.1.S1_s_at & AK098872 & 3.26 & $\begin{array}{l}\text { Chlorophyll a/b-binding protein } \mathrm{CP} 26 \\
\text { precursor - maize }\end{array}$ & AAX95978 (7e-146) \\
\hline Os.37713.1.S1_at & AK070051 & 3.23 & Putative photosystem I antenna protein & BAC83072 (5e-55) \\
\hline Os.28216.2.S1_a_at & AK119545 & 2.59 & $\begin{array}{l}\text { Putative chlorophyll A-B binding protein of } \\
\text { LHCII type III chloroplast precursor (CAB) }\end{array}$ & BAC83393 (2e-153) \\
\hline OsAffx.18836.1.S1_at & $9639 . \mathrm{m} 01283$ & 2.58 & $\begin{array}{l}\text { Chlorophyll a/b-binding protein } \mathrm{CP} 26 \\
\text { precursor - maize }\end{array}$ & AAX95980 (9e-113) \\
\hline Os.5869.3.S1_x_at & CB667246 & 2.02 & $\begin{array}{l}\text { Chlorophyll a/b-binding protein } \mathrm{CP} 24 \\
\text { precursor }\end{array}$ & AAD27882 (8e-78) \\
\hline \multicolumn{5}{|l|}{ Intracellular traffic } \\
\hline Os.2759.1.S1_s_at & AK099409 & 2.46 & $\begin{array}{l}\text { Mitochondrial carnitine/acylcarnitine carrier } \\
\text { putative expressed }\end{array}$ & AAP55124 (3e-116) \\
\hline Os.11654.1.S1_at & AK065544 & 2.26 & SOUL heme-binding protein-like & BAD32927 (8e-174) \\
\hline \multicolumn{5}{|l|}{ Metabolism } \\
\hline Os.11789.1.S1_at & AK099538 & 3.18 & Putative lysine decarboxylase-like protein & AAN61486 (1e-111) \\
\hline Os.26441.2.S1_at & AK119461 & 2.84 & Putative beta-glycosidase & CAD36515 (0.0) \\
\hline Os.7669.1.S1_at & CB643976 & 2.69 & Putative fatty acid elongase & AAR96244 (5e-57) \\
\hline Os.6595.1.S1_a_at & AK062262 & 2.65 & $\begin{array}{l}\text { Magnesium-chelatase subunit } \mathrm{H} \text { family } \\
\text { protein expressed }\end{array}$ & ABF95686 (0.0) \\
\hline Os.14145.1.A1_at & AK070312 & 2.56 & Ferric reductase & BAD18962 (0.0) \\
\hline Os.27713.1.A1_at & AK120567 & 2.54 & Beta-ketoacyl-coA synthase putative expressed & ABA94525 (0.0) \\
\hline Os.12025.1.S1_a_at & AK099966 & 2.43 & Hydrolase/alpha/beta fold family protein & NP_175660 (3e-153) \\
\hline Os.15570.1.S1_at & AK068586 & 2.40 & $\begin{array}{l}\text { Putative glucosyltransferase-3 UDP-glucoronosyl } \\
\text { and UDP-glucosyl transferase }\end{array}$ & BAC83960 (0.0) \\
\hline Os.25109.1.S1_at & AK105536 & 2.35 & Mannan endo-1 4-beta-mannosidase 7 (OsMAN7) & Q2RBB1 (0.0) \\
\hline Os.17814.2.S1_x_at & AK068064 & 2.33 & Putative malate dehydrogenase & BAC20686 (0.0) \\
\hline Os.12839.1.S1_at & AK060512 & 2.26 & Putative beta-keto acyl reductase & BAD22122 (1e-103) \\
\hline Os.26486.1.S1_at & AK120227 & 2.15 & Putative glossy 1 protein/Sterol desaturase & BAD33619 $(0.0)$ \\
\hline OsAffx.27508.100.S1_s_at & AK108972 & 2.15 & Putative methionyl aminopeptidase & BAD08973 (3e-18) \\
\hline Os.9494.1.S1_s_at & CF292249 & 2.14 & $\begin{array}{l}\text { Short-chain dehydrogenase/reductase } \\
\text { SDR family protein }\end{array}$ & NP_001053404 (6e-18) \\
\hline
\end{tabular}


Table 2 (continued)

\begin{tabular}{|c|c|c|c|c|}
\hline Probe set $\mathrm{ID}^{\mathrm{a}}$ & Public ID $^{\mathrm{b}}$ & $\mathrm{FCA}^{\mathrm{c}}$ & Gene description $^{\mathrm{d}}$ & Homolog $(\text { E score })^{\mathrm{e}}$ \\
\hline Os.9724.1.S1_at & AK104714 & 2.14 & $\begin{array}{l}\text { Very-long-chain fatty acid condensing enzyme } \\
\text { putative expressed }\end{array}$ & ABF94686 (0.0) \\
\hline Os.22241.1.S1_s_at & AK099444 & 2.13 & Nitrilase-associated protein putative expressed & ABA98636 (2e-13) \\
\hline Os.14118.1.S1_at & AK099064 & 2.09 & Putative lipase & AAP33477 (3e-100) \\
\hline Os.36960.1.S1_at & AK062283 & 2.08 & Glucosyltransferase NTGT2-like & BAD82532 (0.0) \\
\hline Os.11403.1.S1_at & AK073262 & 2.07 & $\begin{array}{l}\text { Putative N-carbamyl-L-amino acid } \\
\text { amidohydrolase }\end{array}$ & BAD45389 $(0.0)$ \\
\hline Os.8569.1.S1_at & AK060559 & 2.01 & Beta-carotene hydroxylase putative expressed & ABF93742 (9e-98) \\
\hline Os.20755.1.S1_at & AK068040 & 2.13 & $\begin{array}{l}\text { UDP-glucoronosyl/UDP-glucosyl transferase } \\
\text { family protein }\end{array}$ & NP_196793 (2e-87) \\
\hline \multicolumn{5}{|c|}{ - } \\
\hline Os.51579.1.S1_at & AK063100 & 5.03 & No significant similarity found & \\
\hline Os.37849.1.A1_at & AK068289 & 3.73 & Unnamed protein product & BAA90498 (2e-07) \\
\hline Os.17536.1.S1_at & AK063180 & 2.90 & Unknown protein & BAD36443 (3e-63) \\
\hline Os.34400.1.S1_at & AK071545 & 2.61 & $\begin{array}{l}\text { Protein domain with at least } 5 \text { transmembrane } \\
\text { alpha-helices }\end{array}$ & NP_001042652 (2e-102) \\
\hline OsAffx.24703.1.S1_s_at & $9630 . \mathrm{m} 04191$ & 2.60 & No significant similarity found & \\
\hline Os.11944.1.S1_at & AK070758 & 2.53 & No significant similarity found & \\
\hline Os.55256.1.S1_at & AK107743 & 2.51 & Expressed protein & AAP54198 (8e-09) \\
\hline Os.24338.1.A1_at & AK111667 & 2.47 & Os01g0908300 & NP_001045142 (8e-51) \\
\hline Os.5066.1.S1_at & AK073193 & 2.45 & Os01g0219300 & NP_001042419 (4e-44) \\
\hline Os.8395.1.S1_at & AK069514 & 2.31 & Expressed protein & ABA98963 (3e-70) \\
\hline OsAffx.23005.1.S1_x_at & AK108349 & 2.31 & Unknown protein & BAD67880 (2e-17) \\
\hline OsAffx.23005.1.S1_at & AK108349 & 2.30 & Unknown protein & BAD67880 (2e-17) \\
\hline Os.10615.1.S1_x_at & AK058921 & 2.22 & No significant similarity found & \\
\hline Os.10524.2.S1_at & AK060098 & 2.21 & FHA domain containing protein expressed & ABA95708 (4e-83) \\
\hline Os.5648.1.S1_at & AU172533 & 2.21 & No significant similarity found & \\
\hline OsAffx.14410.1.S1_s_at & $9632 . \mathrm{m} 05269$ & 2.13 & Os04g0635000 & NP_001054004 (6e-11) \\
\hline Os.25052.1.S1_at & CA763439 & 2.10 & Hypothetical protein OsJ_000675 & EAZ10850 (2e-23) \\
\hline Os.49304.1.A1_at & AK063613 & 2.08 & Hypothetical protein OsI_013772 & EAY92539 (1e-34) \\
\hline Os.27659.1.S1_x_at & AK069734 & 2.03 & $\begin{array}{l}\text { TMS membrane protein/tumour differentially } \\
\text { expressed protein }\end{array}$ & ABE91594 (4e-133) \\
\hline Os.11190.1.S1_at & AK058995 & 2.01 & Os04g0402700/Hypothetical protein & NP_001052695 (7e-28) \\
\hline \multicolumn{5}{|l|}{ Protein synthesis/turnover } \\
\hline Os.11707.1.A1_at & CB656443 & 4.46 & Putative cysteine proteinase & AAM34401 (7e-37) \\
\hline \multicolumn{5}{|l|}{ Secondary metabolism } \\
\hline Os.9685.1.S1_a_at & AK067949 & 2.51 & Putative cinnamoyl CoA reductase & BAD28656 (2e-171) \\
\hline Os.46551.1.S1_at & AK064736 & 2.31 & Putative chalcone flavonoid $3^{\prime}$ - hydroxylase & AAN04937 (0.0) \\
\hline \multicolumn{5}{|l|}{ Signal transduction } \\
\hline OsAffx.16737.1.S1_at & AK067579 & 3.35 & $\begin{array}{l}\text { Phototropic-responsive protein putative/a } \\
\text { light-activated serine-threonine protein kinase }\end{array}$ & NP_187478 (3e-31) \\
\hline Os.12110.1.S1_at & AK064985 & 2.88 & $\begin{array}{l}\text { RPT2 expressed /nonphototropic hypocotyl } \\
1 \text { (NPH1)/a serine-threonine protein kinase }\end{array}$ & ABA91174 (0.0) \\
\hline Os.55822.1.A1_at & AK108850 & 2.39 & $\begin{array}{l}\text { Putative serine/threonine protein phosphatase } \\
\text { 2A (PP2A) regulatory subunit B' }\end{array}$ & BAD67828 (1e-95) \\
\hline Os.7676.1.S1_at & AY476807 & 2.30 & Extracellular calcium sensing receptor & AAS00828 (1e-146) \\
\hline Os.7140.1.S1_at & AK061014 & 2.28 & $\begin{array}{l}\text { GUN4-like family protein expressed/regulator } \\
\text { of chlorophyll synthesis and intracellular } \\
\text { signaling }\end{array}$ & ABA92587 (4e-110) \\
\hline Os.45531.1.S1_at & CB621884 & 2.19 & Receptor-like kinase & AAF78017 (1e-48) \\
\hline Os.7304.1.S1_at & AK069537 & 2.16 & Putative protein kinase & BAB92217 (0.0) \\
\hline Os.28255.1.S1_at & AK070519 & 2.07 & $\begin{array}{l}\mathrm{ABC} 1 \text { family protein } / \mathrm{Mn} 2+\text {-dependent } \\
\text { serine/threonine protein kinase }\end{array}$ & NP_565025 (0.0) \\
\hline \multicolumn{5}{|c|}{ 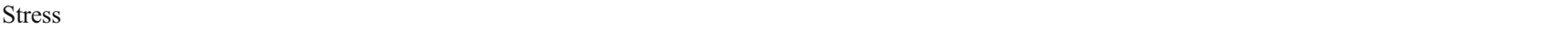 } \\
\hline Os.53572.1.S1_at & AK073356 & 3.66 & Universal stress protein USP1-like protein & BAC16006 (2e-84) \\
\hline Os.37549.1.S1_at & AK102998 & 3.35 & Auxin/aluminum-responsive protein putative & NP_197415 (3e-10) \\
\hline Os.15692.1.S1_at & AK069017 & 2.68 & Cytochrome P450 family protein expressed & ABF97430 (0.0) \\
\hline
\end{tabular}


Table 2 (continued)

\begin{tabular}{|c|c|c|c|c|}
\hline Probe set ID ${ }^{\mathrm{a}}$ & Public $\mathrm{ID}^{\mathrm{b}}$ & $\mathrm{FCA}^{\mathrm{c}}$ & Gene description $^{\mathrm{d}}$ & Homolog $(\text { E score })^{\mathrm{e}}$ \\
\hline Os.5377.1.S1_at & AK105891 & 2.52 & $\begin{array}{l}\text { Selenium-binding protein-like (pentatricopeptide } \\
\text { repeat-containing protein) }\end{array}$ & BAD07861 (1e-152) \\
\hline Os.34316.1.S1_at & AK071882 & 2.37 & $\begin{array}{l}\text { Hypothetical protein OsJ_021319/DnaJ } \\
\text { domains }\end{array}$ & EAZ37836 (9e-21) \\
\hline Os.17037.1.S2_a_at & CB671899 & 2.20 & $\begin{array}{l}\text { Putative Altered Response to Gravity/DnaJ } \\
\text { domain }\end{array}$ & BAD73072 (7e-21) \\
\hline Os.35448.1.S1_at & AK067480 & 2.20 & Thioredoxin h-like protein & AAN63618 (4e-17) \\
\hline \multicolumn{5}{|l|}{ Transcription } \\
\hline Os.46600.1.S1_at & AK058809 & 3.77 & $\begin{array}{l}\text { Helix-loop-helix DNA-binding domain } \\
\text { containing protein }\end{array}$ & AAP53429 (7e-113) \\
\hline Os.56880.1.S1_at & AK110526 & 3.66 & $\begin{array}{l}\text { Basic-leucine zipper (bZIP) transcription } \\
\text { factor domain containing protein }\end{array}$ & NP_001043273 (5e-39) \\
\hline Os.31716.2.A1_at & $\mathrm{BI} 305433$ & 3.03 & Scl1 protein/GRAS family transcription factor & AAF00139 (2e-24) \\
\hline Os.49848.1.S1_at & AK111960 & 2.71 & Putative MYB-related protein 1 & BAD07916 (4e-71) \\
\hline Os.25588.2.S1_x_at & AK101674 & 2.67 & Putative MYB protein & BAC79723 (5e-88) \\
\hline Os.3386.1.S1_x_at & AK062487 & 2.63 & MYB protein & BAD36195 (7e-26) \\
\hline Os.27085.1.A1_at & CB656188 & 2.34 & $\begin{array}{l}\text { Putative typical P-type R2R3 MYB protein/SANT } \\
\text { domain containing protein }\end{array}$ & BAD10148 (2e-12) \\
\hline Os.46081.1.S1_at & AK060177 & 2.18 & $\begin{array}{l}\text { Hypothetical protein OsJ_029817/transcription } \\
\text { factor activity }\end{array}$ & EAZ15608 (2e-96) \\
\hline Os.16025.1.S1_s_at & CA764046 & 2.14 & Putative bZIP protein HY5 & BAD32844 (1e-07) \\
\hline Os.19539.1.S1_at & AK058932 & 2.04 & Putative DNA helicase & BAD08079 (0.0) \\
\hline Os.11409.1.S1_at & AK063523 & 2.04 & Basic helix-loop-helix putative expressed & ABF93851 (6e-36) \\
\hline Os.17902.1.S1_at & AK111634 & 2.03 & MYB17 protein & CAD44611 (7e-34) \\
\hline Os.26437.1.A1_s_at & CB673064 & 2.02 & bZIP transcription factor family protein expressed & ABA99796 (6e-51) \\
\hline Os.23030.1.S1_at & AK060509 & 2.05 & $\begin{array}{l}\text { Arabidopsis NAC domain containing protein } 83 \text {; } \\
\text { transcription factor }\end{array}$ & NP_196822 (4e-24) \\
\hline \multicolumn{5}{|c|}{ 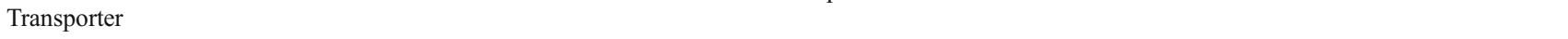 } \\
\hline Os.11804.1.S1_at & CB624264 & 3.27 & $\begin{array}{l}\text { Tryptophan/tyrosine permease family } \\
\text { protein expressed . }\end{array}$ & ABF95016 (3e-111) \\
\hline Os.52259.1.S1_at & AK065945 & 2.55 & Major Facilitator Superfamily protein expressed & ABA91097 (0.0) \\
\hline Os.5210.1.S1_at & AK072183 & 2.49 & Putative sodium-dicarboxylate cotransporter & BAD09278 (0.0) \\
\hline Os.19896.2.S1_x_at & CB623166 & 2.47 & Putative sodium transporter & CAD37186 (1e-64) \\
\hline Os.41637.1.S1_at & CA766063 & 2.40 & MDR-like ABC transporter & CAD59585 (8e-14) \\
\hline Os.27393.1.S1_s_at & AK061521 & 2.39 & MDR-like ABC transporter & CAD59590 (0.0) \\
\hline Os.57530.1.S1_x_at & AJ491855 & 2.27 & Putative sodium transporter & CAD37198 (0.0) \\
\hline Os.25449.1.S1_at & AK108531 & 2.21 & Integral membrane transporter family protein & NP_180886 (1e-62) \\
\hline Os.17299.1.S1_at & CB655994 & 2.18 & Putative glycerol 3-phosphate permease & AAS82603 (7e-112) \\
\hline Os.52960.1.S1_x_at & AK072316 & 2.17 & Putative amino acid permease & AAP46198 (3e-146) \\
\hline Os.17681.1.S1_at & AK072059 & 2.14 & Monosaccharide transporter 1 & BAB19862 (4e-158) \\
\hline Os.21635.1.S1_at & AF543419 & 2.11 & Major facilitator superfamily antiporter & AAN33182 (0.0) \\
\hline Os.52621.1.S1_x_at & AK068351 & 2.04 & Nitrate transporter putative & AAT85061 (8e-162) \\
\hline \multicolumn{5}{|l|}{ Transposons } \\
\hline Os.16593.1.S1_at & BI803439 & 2.14 & $\begin{array}{l}\text { Retrotransposon protein putative Ty3-gypsy } \\
\text { subclass expressed }\end{array}$ & ABA92095 (1e-34) \\
\hline Os.24273.1.A1_at & AK102986 & 2.07 & $\begin{array}{l}\text { Hypothetical protein osi_009106/transposase } \\
\text { activity }\end{array}$ & EAY87873 (5e-30) \\
\hline
\end{tabular}

${ }^{\text {a }}$ Affymetrix Probe set ID of downregulated gene

${ }^{b}$ The public representative ID of these genes obtained from Affymetrix NetAffx analysis center

${ }^{\mathrm{c}}$ PLIER normalized absolute fold change value for downregulation

${ }^{\mathrm{d}}$ The putative function of the gene based on BLASTX analysis

${ }^{\mathrm{e}}$ Accession ID of the homologous protein based upon which the function has been assigned. The corresponding BLASTX E- score is shown in brackets

${ }^{\mathrm{f}}$ Genes encoding hypothetical proteins or those with no significant matches in the databases are kept in this category 
Table 3 List of Cellulase Responsive Rice Genes Associated with Jasmonic-Acid-Mediated Defense Pathway

\begin{tabular}{|c|c|c|c|c|}
\hline Probe set $\mathrm{ID}^{\mathrm{a}}$ & Public ID ${ }^{b}$ & $\mathrm{FCA}^{\mathrm{c}}$ & Gene description $^{\mathrm{d}}$ & Homolog $(\text { E score })^{\mathrm{e}}$ \\
\hline OsAffx.17366.1.S1_at ${ }^{\mathrm{f}}$ & $9636 . \mathrm{m} 03709$ & 6.54 & Ethylene-binding protein-AP2 domain containing & BAD38371 (4e-41) \\
\hline Os.6043.1.S1_at & AB040744.1 & 5.30 & $\begin{array}{l}\text { RERJ1(Helix-loop-helix domain containing } \\
\text { transcriptin factor) }\end{array}$ & AB040744 (8e-111) \\
\hline Os.15849.1.S1_s_at ${ }^{\mathrm{f}}$ & AK062882.1 & 4.34 & AP2 domain-containing transcription factor-like & BAD17116 (4e-15) \\
\hline Os.55259.1.S1_at & AK107750.1 & 3.77 & ZIM motif family protein, expressed & ABF94311 (1e-07) \\
\hline Os.4671.1.S1_a_at & AF435970.1 & 3.47 & Jacalin lectin family protein, putative & P24120 (2e-78) \\
\hline Os.46849.1.S1_at & AK107854 & 3.46 & ZIM motif family protein, expressed & AAP53563 (7e-23) \\
\hline Os.9923.1.S1_s_at & AK120087.1 & 2.58 & ZIM motif family protein, expressed & ABF94311 (9e-57) \\
\hline Os.8741.1.S1_at & AK102303.1 & 2.57 & $\begin{array}{l}\text { Poly polymerase catalytic domain containing } \\
\text { protein/RADICAL-INDUCED CELL DEATH1 }\end{array}$ & ABF94777 (0.0) \\
\hline Os.35343.1.A1_at & AK067922.1 & 2.51 & No apical meristem protein (NAC domain containing) & ABA95706 (2e-116) \\
\hline Os.10356.1.S1_at & AK073589.1 & 2.09 & ZIM motif family protein, expressed & ABF94310 (2e-82) \\
\hline Os.50513.1.S1_at & AK121554.1 & 2.05 & Putative 12-oxophytodienoate reductase (OPR2) & BAD61319 $(0.0)$ \\
\hline Os.12012.1.S1_at & AK061602.1 & 1.97 & ZIM motif family protein, expressed & AAP53568 (5e-83) \\
\hline Os.22000.1.S1_at & AK061042.1 & 1.95 & Endochitinase/ putative PR3 like & BAA03749 (9e-166) \\
\hline Os.8778.1.S1_a_at & AB040743.1 & 1.63 & Cis-12-oxo-phytodienoic acid-reductase 1 (OPR1) & BAD26703 (0.0) \\
\hline Os.3415.1.S1_s_at & AB016497.1 & 1.51 & Chitinase/ putative PR3 like & BAA31997 (2e-135) \\
\hline Os.46443.1.S1_at & AY536428.1 & 1.46 & $\begin{array}{l}\text { MYC protein/ATMYC2 (JASMONATE INSENSITIVE 1) } \\
\text { ortholog }\end{array}$ & AAS66204 (0.0) \\
\hline
\end{tabular}

${ }^{a}$ Affymetrix Probe set ID

${ }^{\mathrm{b}}$ The public representative ID obtained from Affymetrix NetAffx analysis center

${ }^{\mathrm{c}}$ PLIER normalized absolute fold change value

${ }^{\mathrm{d}}$ The putative function of the gene based on BLASTX analysis

${ }^{\mathrm{e}}$ Accession ID of the homologous protein based upon which the function has been assigned. The corresponding BLASTX E score is shown in brackets

${ }^{\mathrm{f}}$ These are two probe set IDs for the same gene

Validation of differentially expressed biologically significant genes by real-time PCR analysis

The expression profile of a few selected biologically significant genes was also examined by SYBR green based realtime PCR. Primers were designed to amplify $130-170$ bp gene fragments of eight upregulated genes (OsAP2/ERF, OsRERJ1, OSMYC, OSOPR2, OsAOS, OSCOPINE1, OsSGT, and $O s B S M T 1)$ and one downregulated gene (OsMtN3; a host susceptibility factor; see Supplementary Table S2; for list of primers). The results indicate that $O S A P 2 / E R F$, OsRERJ1, OSMYC, OsOPR2, OsAOS, OsCOPINE1, OSSGT, and OsBSMT1 are upregulated while the OsMtN3 gene is down regulated following ClsA treatment (Fig. 2).

Wild-type $X$. oryzae pv. oryzae suppressed ClsA-induced expression of OSAP2/ERF and OSRERJ1

Rice seedlings were infiltrated with either ClsA or ClsA along with wild-type $X$. oryzae pv. oryzae in order to determine if the ClsA-induced expression of OsAP2/ERF, and OsRERJ1 is suppressed by co-treatment with the bacterium. Expression of these genes was analyzed by SYBR green-based quantitative real-time PCR in three independent biological replicates. The relative fold change was calculated in comparison to their expression in buffer-treated leaves and the $O S G A P D H$ gene as an endogenous control. Although there was variability between individual experiments, overall, the results indicated that co-treatment with wild-type $X$. oryzae pv. oryzae suppressed the induction of expression of OsAP2/ERF and OsRERJ1 by ClsA at significance level $p<0.05$ (Table 4).

\section{Sequence features of $O S A P 2 / E R F$}

The OSAP2 (APETALA2)/ERF gene that is upregulated following ClsA treatment is an 849 bp intron less gene encoding a 282 amino acid protein. Sequence analysis of the OSAP2/ERF using prosite (http://www.expasy.ch/prosite) has revealed that it contains a single AP2/ERF domain which spans from 138-195 amino acid residues (Supplementary Fig. S2). The presence of A and D amino acid at position 151 and 156 is a characteristic of AP2/ERF proteins which distinguishes them from related DREB proteins wherein the $\mathrm{V}$ and $\mathrm{E}$ amino acids are present at the corresponding positions (Sakuma et al. 2002). AP2/ERF domain has two sub-domains: YRG, involved in DNA binding and RAYD, 


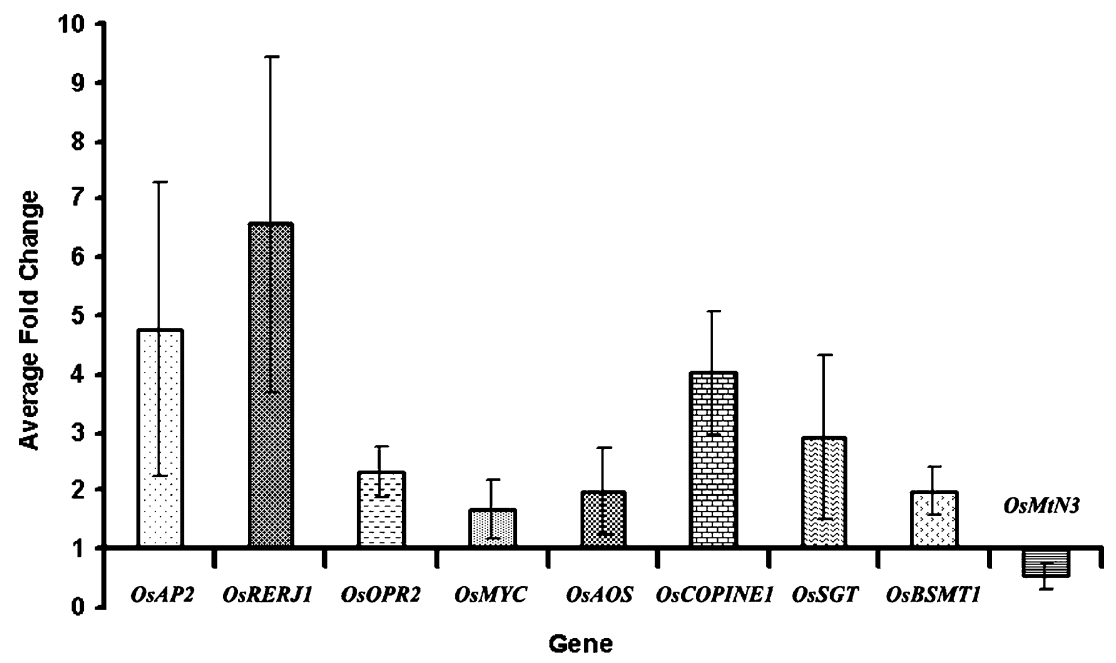

Fig. 2 Real-time PCR analysis of some differentially expressed genes. Total RNA was isolated from either ClsA- or buffer-treated rice leaves; first-strand cDNA was synthesized, and gene expression was assayed by SYBR green-based real-time PCR. The genes that were assayed are the following: OsAP2 (AP2/ERF transcription factor), OsRERJ1 (methyl JA induced helix loop helix rice transcription factor), OsOPR2 (a 12-oxophytodienoate reductase), OsMYC (a key transcription factor in the JA-mediated defense pathway), OsAOS (Allene Oxide Synthase), OsCOPINE1, OsSGT (salicylic acid gluco-

which is probably involved in DNA binding through the interaction of its hydrophobic phase with the major groove of DNA (Okamuro et al. 1997). The protein also contains nuclear localization signal sequences. The protein is predicted to contain two $\alpha$ helices and four $\beta$ sheets. Amino acid residues in the pink box are predicted to be in direct contact with DNA (Allen et al. 1998; Nakano et al. 2006). Besides this, presence of a putative zinc finger motif $(\mathrm{C} \times 2 \mathrm{C} \times 4 \mathrm{C} \times 2 \sim 4 \mathrm{C})$ on the $\mathrm{N}$-terminal region of the protein suggests its involvement in DNA binding or protein-protein interactions. Due to presence of this motif, the protein has been previously classified to be a member of $\mathrm{Xb}$ subgroup of AP2/ERF proteins of rice (Nakano et al. 2006). syltransferase), OSBSMT1 (benzothiadiazole-induced $S$-adenosyl-L methionine:salicylic acid carboxyl methyltransferase1), and OsMtN3 (Os8N3; a host susceptibility factor). The relative fold changes were calculated by using $2^{- \text {ddC }} \mathrm{T}$ method using the expression of buffer treated leaves as a comparison. The OsGAPDH (Glyceraldehyde-3phosphate dehydrogenase) gene was used as an endogenous control. Mean and standard deviation from at least four biological replicates for each gene are presented.

Agrobacterium-mediated transient transfer of $O s A P 2 / E R F$ induces defense responses such as a PCD reaction in rice roots and callose deposition in leaves

PCD is a form of plant defense response (Pennell and Lamb 1997). We treated rice roots with Agrobacterium strain LBA4404/pOsAP2/ERF and stained with propidium iodide (PI) followed by confocal microscopy in order to determine if transient transfer of $O S A P 2 / E R F$ induces PCD. Cell-wallassociated fluorescence was observed in roots treated with Agrobacterium strain LBA4404/pSB11; very few cells took up PI stain, and the distribution of the stain indicated that the nucleus remained compact (Fig. 3). In contrast, the PI

Table 4 Suppression of Cellulase-induced Expression of OsRERJ1 and OsAP2/ERF Transcription Factor Genes by Wild-type X. oryzae pv. oryzae

\begin{tabular}{|c|c|c|c|c|c|c|}
\hline \multirow[t]{2}{*}{ Genes $^{\mathrm{a}}$} & \multicolumn{2}{|c|}{ Experiment- $1^{\mathrm{b}}$} & \multicolumn{2}{|c|}{ Experiment $-2^{b}$} & \multicolumn{2}{|c|}{ Experiment- $3^{\mathrm{b}}$} \\
\hline & $\mathrm{ClsA}^{\mathrm{c}}$ & $\mathrm{ClsA}+\mathrm{Xoo}^{\mathrm{c}}$ & $\mathrm{ClsA}^{\mathrm{c}}$ & $\mathrm{ClsA}+\mathrm{Xoo}^{\mathrm{c}}$ & $\mathrm{ClsA}^{\mathrm{c}}$ & $\mathrm{ClsA}+\mathrm{Xoo}^{\mathrm{c}}$ \\
\hline OsAP2/ERF & 5.56 & 1.33 & 5.11 & 1.43 & 2.64 & 0.66 \\
\hline OsRERJI & 8.26 & 1.48 & 9.45 & 1.7 & 5.86 & 1.36 \\
\hline
\end{tabular}

${ }^{a}$ Rice genes upregulated following cellulase treatment

${ }^{\mathrm{b}}$ Fifteen days old rice seedlings were infiltrated with either cellulase (ClsA) or cellulase along with wild type X. oryzae pv. oryzae (ClsA+Xoo). Buffer-treated leaves were used as control. Total RNA was isolated from each of the samples, converted into cDNA and subjected to real-time PCR analysis

${ }^{\mathrm{c}}$ The relative fold changes were calculated by $2^{-\mathrm{ddC}} \mathrm{T}$ method in comparison to expression in buffer treated leaves. The $O s G A P D H$ (Glyceraldehyde-3-phosphate dehydro-genase) gene was used as an endogenous control 

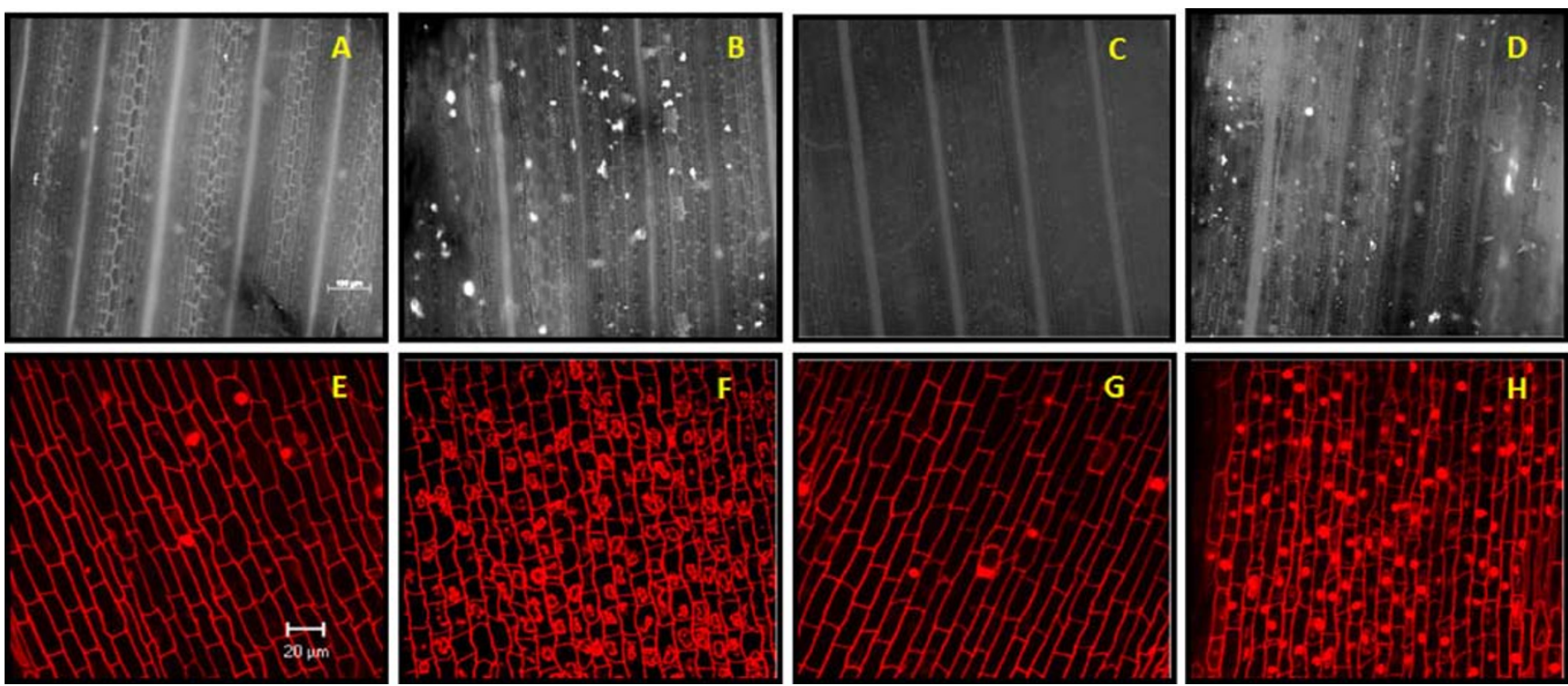

Fig. 3 Transient transfer of $O s A P 2 / E R F$ induces callose deposition in rice leaves and cell death in rice roots. Rice leaves were syringe infiltrated with one of the following: water, ClsA, LBA4404/ pSB11, and $L B A 4404 / p O s A P 2 / E R F$. After $15 \mathrm{~h}$, they were stained with aniline blue and examined under epifluorescence microscope. White spots in these pictures are indicative of callose deposition. $\mathbf{b}$ cellulase and $\mathbf{d}$ $L B A 4404 / p O s A P 2 / E R F$ induce increased callose deposition compared with either a water or $\mathbf{c}$ LBA4404/pSB11. Rice roots were also treated

either with water, cellulase, LBA4404/pSB11, LBA4404/pOsAP2/ERF and stained with propidium iodide (PI), and examined under a confocal microscope. Treatment with either water (e) or LBA4404/ pSB11 (g) did not induce cell death (intake of PI), whereas treatment with cellulase (f) and $L B A 4404 / p O s A P 2 / E R F(\mathbf{h})$ induced cell death and extensive nuclear fragmentation. Similar results were obtained in at least three independent experiments.

stain was taken up by all the cells in rice roots treated with LBA4404/pOsAP2/ERF indicating that extensive cell death has been induced (Fig. 3). As expected, cell death was not observed in roots treated with water, and extensive cell death was observed in roots treated with ClsA. Syringe infiltration of rice leaves with LBA4404/pOsAP2/ERF strain but not LBA4404/pSB11 strain induced callose deposition in rice leaves. As expected, infiltration with ClsA induced callose deposition while only background levels of callose deposition were observed in leaves infiltrated with water (Fig. 3, Table 5).
Prior treatment with LBA4404/pOsAP2/ERF strain induces resistance to subsequent $X$. oryzae pv. oryzae infection in rice

The midveins of leaves from 40-day-old rice plants were preinjected with one of the following: water and saturated culture of LBA4404/pSB11 and LBA4404/pOsAP2/ERF. All leaves were subsequently infected with $X$. oryzae pv. oryzae. Across four independent experiments, all the leaves $(100 \%)$ that had been preinjected with water exhibited lesions induced by $X$. oryzae pv. oryzae. About $72-77 \%$ of the

Table 5 Transient Transfer of OsAP2/ERF Induces Callose Deposition in Rice Leaves

Mean number of callose deposits $/ 0.6 \mathrm{~mm}^{2}$ area $\pm \mathrm{SD}^{\mathrm{a}}$

\begin{tabular}{lllll}
\hline Exp $^{\mathrm{b}}$ & Water & Cellulase & LBA4404/pSB11 & LBA4404/pOsAP2/ERF \\
\hline 1 & $13.30 \pm 4.08$ & $141.20 \pm 37.43$ & $23.10 \pm 10.07$ & $87.00 \pm 15.29$ \\
2 & $11.70 \pm 3.09$ & $154.00 \pm 37.21$ & $29.00 \pm 15.81$ & $97.50 \pm 35.73$ \\
3 & $14.40 \pm 3.50$ & $126.10 \pm 29.17$ & $25.50 \pm 6.43$ & $80.00 \pm 16.47$
\end{tabular}

\footnotetext{
${ }^{a}$ Rice leaves were syringe infiltrated with one of the following: water, cellulase $\left(100 \mu \mathrm{g} \mathrm{ml}{ }^{-1}\right), \mathrm{LBA} 4404 / \mathrm{pSB} 11$, and LBA4404/pOSAP2/ERF, stained with aniline blue, and examined under epifluorescence microscope. Mean and standard deviation were calculated for number of callose deposits from a leaf area of $0.60 \mathrm{~mm}^{2}$

${ }^{\mathrm{b}}$ Data were collected from at least ten leaves in each experiment. ANOVA was performed, and the values obtained after infiltration with LBA4404/pOsAP2/ERF were found to be significantly different $(p<0.05)$ as compared to all other treatments
} 
Table 6 Transient Transfer of OsAP2/ERF Induces Resistance Against Xanthomonas oryzae pv. oryzae Infection

\begin{tabular}{|c|c|c|c|c|c|c|c|c|}
\hline \multirow[t]{2}{*}{ Treatments $^{\mathrm{a}}$} & \multicolumn{4}{|c|}{$\begin{array}{l}\% \text { of infected leaves (no. of infected leaves/total no. } \\
\text { of leaves) })^{\mathrm{b}}\end{array}$} & \multicolumn{4}{|c|}{$\begin{array}{l}\text { Mean lesion length of leaves showing disease symptoms } \\
(\mathrm{cm}) \pm \mathrm{SD}^{\mathrm{c}}\end{array}$} \\
\hline & Exp 1 & Exp 2 & Exp 3 & Exp 4 & Exp 1 & Exp 2 & Exp 3 & Exp 4 \\
\hline Water & $100(19 / 19)$ & $100(28 / 28)$ & $100(21 / 21)$ & $100(25 / 25)$ & $11.2 \pm 7.01$ & $10.50 \pm 6.42$ & $11.86 \pm 4.82$ & $10.18 \pm 3.66$ \\
\hline LBA4404/ pSB11 & $72(18 / 25)$ & $75(21 / 28)$ & $77(14 / 18)$ & $76(19 / 25)$ & $10.8 \pm 7.36$ & $9.38 \pm 5.93$ & $7.71 \pm 5.30$ & $8.68 \pm 5.75$ \\
\hline LBA4404/pOsAP2/ERF & $30(7 / 23)$ & $50(10 / 20)$ & $50(9 / 18)$ & $48(12 / 25)$ & $3.57 \pm 1.51$ & $5.0 \pm 1.56$ & $3.56 \pm 1.24$ & $5.58 \pm 1.73$ \\
\hline
\end{tabular}

${ }^{a}$ Midveins of leaves of 40 days old rice plants (TN-1) were preinjected with either water alone, or water containing $\sim 10^{9}$ cells of LBA4404/ pSB11 or LBA4404/pOsAP2/ERF. After $6 \mathrm{~h}$, Xanthomonas oryzae pv. oryzae was inoculated onto the midvein, 2 to $3 \mathrm{~cm}$ below the point of initial injection, by pricking with a needle that had been used to touch a freshly grown colony of Xanthomonas oryzae pv. oryzae. Lesion lengths were measured 10 days after inoculation

${ }^{\mathrm{b}}$ 18-28 leaves were used in each experiment and four independent replications were performed

${ }^{\mathrm{c}}$ Lesion lengths were measured 10 days after inoculation. Mean and standard deviation were calculated for each of the treatments. ANOVA was performed and the values obtained after treatment with LBA4404/pOsAP2/ERF were found to be significantly different $(p<0.05)$ as compared to all other treatments

leaves which were preinjected with LBA4404/pSB11 exhibited $X$. oryzae pv. oryzae-induced lesions. Interestingly, only $30-50 \%$ of leaves that were preinjected with LBA4404/ pOSAP2/ERF exhibited disease lesions (Table 6). Among the leaves that exhibited disease lesions, the lesion lengths were $3.56 \pm 1.24$ to $5.58 \pm 1.73 \mathrm{~cm}, 7.71 \pm 5.30$ to $10.8 \pm 7.36 \mathrm{~cm}$, and $10.18 \pm 3.66$ to $11.86 \pm 4.82 \mathrm{~cm}$ long following preinjection with $\mathrm{LBA} 4404 / \mathrm{p} O s A P 2 / E R F$, LBA4404/pSB11, and water, respectively.

\section{Discussion}

$X$. oryzae pv. oryzae secretes a cocktail of at least five cellwall-degrading enzymes, including ClsA, to damage the plant cell wall during infection. Besides these five enzymes, the $X$. oryzae pv. oryzae genome additionally encodes five cellulases, three xylanases, one pectinase as well as several other categories of cell-wall-degrading enzymes (Lee et al. 2005). It is possible that many of these enzymes are expressed only during in planta growth, and it is likely that the action of each one of these enzymes will induce rice innate immune responses. In spite of the apparent importance of cell-wall-degrading enzymes for induction of host innate immunity (Jha et al. 2007), very little information is available about plant functions that participate in the elaboration of innate responses that are induced following their action.

To address this lacuna, we have performed transcriptional profiling of rice gene expression changes that occur following cell-wall damage initiated by treatment with $X$. oryzae pv. oryzae ClsA. In our experiments, we have infiltrated $5 \mu \mathrm{g}$ of ClsA protein into approximately $1 \mathrm{~cm}^{2}$ of leaf area although we find that infiltration of even $4 \mu \mathrm{g}$ of enzyme is sufficient to induce a HR like response. In the laboratory, approximately $4 \mu \mathrm{g}$ of ClsA is secreted per milliliter of a saturated culture (approx. $1 \times 10^{9}$ cell) of $X$. oryzae pv. oryzae. During infection, $1 \mathrm{~cm}^{2}$ of leaf area supports $1 \times 10^{9}$ cells of $X$. oryzae pv. oryzae, and we expect that these cells would secrete about $4 \mu \mathrm{g}$ of ClsA. As a number of other cell-wall-degrading enzymes, besides ClsA, are secreted by $X$. oryzae pv. oryzae, we expect that the total amounts of cell-wall-degrading enzymes secreted by $X$. oryzae pv. oryzae is going to be much higher than that used in this experiment. Besides the above, there is evidence that secretion by $\mathrm{T} 2 \mathrm{~S}$ of $V$. cholerae and $P$. aeruginosa occurs in a polar manner because the T2S apparatus is localized to one pole of the cell, possibly at the point of attachment to host surface (Scott et al. 2001; Senf et al. 2008). If the T2S of $X$. oryzae pv. oryzae is similarly localized, the local concentrations of cell-wall-degrading enzymes are likely to be quite high during infection.

We have performed homology searches using BLASTX for each of the 267 genes that were found to be differentially expressed $(\geq 2$-fold at $p<0.05$ ) following ClsA treatment. A striking observation is that a large number of putative transcription factors (26 upregulated and 15 downregulated) are differentially expressed following ClsA treatment. This is suggestive of global changes in rice gene expression during the HR like reaction. The microarray analysis has revealed the downregulation of genes for several chlorophyll $a / b$ binding proteins and suppression of the rice ortholog of GUN4, a critical activator of chlorophyll synthesis in Arabidopsis (Larkin et al. 2003). The gene ontology enrichment analysis also indicated that functions involved in photosynthesis are downregulated. This suggests an overall downregulation of synthesis of chlorophyll and chlorophyll-binding proteins during ClsA-induced cell death and a possible reduction in photosynthetic efficiency during this process. It was also interesting to note that the gene which exhibits maximum upregulation in our microarray is 
homologous to a gene encoding a senescence associated protein of Arabidopsis (AT1G74940.1) suggesting a possible correlation between some events associated with senescence and HR. Three condensing enzymes and a ß-ketoacyl reductase that are homologous to Arabidopsis functions that participate in cuticle development were also downregulated following ClsA treatment. Defects in cuticle development have been shown to cause enhanced resistance to Botrytis cinareae and enhanced disease symptoms in response to an avirulent strain of Pseudomonas syringae pv. tomato in Arabidopsis thaliana (Chassot et al. 2007; Bessire et al. 2007; Tang et al. 2007).

We had earlier demonstrated that co-treatment with wildtype $X$. oryzae pv. oryzae results in suppression of ClsAinduced rice defense responses. We demonstrate here, using real-time PCR, that co-treatment with wild-type $X$. oryzae pv. oryzae also results in suppression of ClsA-induced expression of the rice transcription factor genes OsAP2/ERF and OsRERJ1. AP2/ERF members are downstream components of both the ethylene and jasmonatic acid (JA) pathways and are key to the integration of both signals (Lorenzo et al. 2003; Zhang et al. 2004a, b). The OsRERJ1 gene is upregulated by JA (Kiribuchi et al. 2004). Chini et al. (2007) and Thines et al. (2007) have demonstrated that several Arabidopsis ZIM family transcription factors serve as negative regulators of the JA pathway through repression of a MYC transcription factor (JASMONATE INSENSITIVE1, JIN1; Lorenzo et al. 2004), which is the key activator of JA responsive genes. JA promotes ubiquitination and degradation of these ZIM transcription factors, resulting in expression of MYC and activation of the JA responsive genes. Transcription of the genes for these ZIM transcription factors is upregulated by JA, and this has been attributed to serve in damping the JA pathway (Chini et al. 2007; Thines et al. 2007). The microarray data presented here indicates that rice homologs of these ZIM transcription factors and OSMYC (or OsJINI) are upregulated by ClsA treatment. Besides the above, we have found that several genes that encode functions predicted to be involved in JA biosynthesis are upregulated following ClsA treatment. These results, taken together, suggest that the JA-mediated pathway might be associated with ClsA-induced HR. This needs to be confirmed using rice lines that are either mutated or knocked down for the JA pathway.

Nakano et al. (2006) indicate that the genome sequences of Arabidopsis and rice contain 122 and 139 ERF proteins, respectively, which are subdivided into I to $\mathrm{X}$ subgroups. Our sequence analysis indicates that the OSAP2/ERF studied here belongs to $\mathrm{Xb}$ subgroup of the ERF subfamily which is characterized by the presence of Cys repeats at its N-terminal region. We have treated rice leaves and roots with LBA4404/ pOSAP2/ERF in order to assess the effect of transient transfer of $O S A P 2 / E R F$ on induction of innate immune responses.
Rice leaves infiltrated with LBA4404/pOsAP2/ERF showed a significant increase in callose deposition as compared to leaves treated with LBA4404/pSB11 (control). Similarly, rice roots treated with LBA4404/pOsAP2/ERF exhibit programmed cell death, which is absent in roots treated with LBA4404/pSB11. Prior injection of LBA4404/pOsAP2/ERF in rice mid-veins induces resistance against subsequent infection with $X$. oryzae pv. oryzae. Treatment with LBA4404/pSB11 also induces a certain degree of resistance against subsequent bacterial infection as evident from fewer leaves showing $X$. oryzae pv. oryzae induced disease lesions. Although this resistance is significantly lesser than that induced by LBA4404/pOsAP2/ERF, it suggests that Agrobacterium can also induce a certain amount of resistance. Overall, these results suggest that transient overexpression of $O S A P 2 / E R F$ induces rice resistance responses. However, these results need to be confirmed by making stably transgenic rice lines that overexpress $O S A P 2 / E R F$, preferably under the control of an inducible promoter.

Besides the cell-wall-degrading enzymes secreted by $X$. oryzae pv. oryzae, several other molecules are known to be elicitors of innate immune responses in rice. The OsFLS2 protein, a receptor kinase, has been shown to be the receptor for the flg22 peptide (Takai et al. 2008); Xa21, a rice resistance protein and receptor kinase, is the receptor for AvrXa21 peptide (Lee et al. 2009), and the rice chitinbinding protein $\mathrm{CeBiP}$, a transmembrane protein with particular extracellular motifs, is the receptor for chitin (Kaku et al. 2006). The small GTPase OsRAC1 is involved in the immune responses induced by $\mathrm{N}$-acetylchitooligosaccharide and spingolipids (Nakashima et al. 2008). None of these above mentioned proteins were differentially expressed (2-fold level) in the microarray experiments described here. However, several putative receptor-like protein kinases are differentially expressed following ClsA treatment, and it remains to be determined whether these are involved either in elicitor perception or in further signal processing. $X$. oryzae pv. oryzae lipopolysaccharide is also an inducer of rice defense responses, but the receptor has not yet been characterized (Desaki et al. 2006).

In summary, we have performed microarray analysis of gene expression changes that are induced in rice by $\mathrm{ClsA}$ treatment. A number of rice functions, particularly transcription factors, are differentially expressed under these conditions. Initial studies using Agrobacterium-mediated transient gene transfers suggest that overexpression of one of the highly upregulated transcription factor genes, $O s A P 2 / E R F$, results in induction of callose deposition, cell death, and enhanced resistance against subsequent infection by Xanthomonas oryzae pv. oryzae. Future studies will be aimed at understanding the role of OSAP2/ERF and other differentially expressed genes in ClsA-induced innate immunity in rice. 


\section{Methods}

\section{RNA isolation}

Fifteen-day-old seedlings of the susceptible rice cultivar TN-1 were grown in a greenhouse in the months of December/ January and shifted to a growth chamber $\left(28^{\circ} \mathrm{C} ; 80 \%\right.$ relative humidity; light intensity of $440 \mu \mathrm{mole} / \mathrm{m}^{2} / \mathrm{s} ; 12 / 12 \mathrm{~h}$ light/dark cycle) $48 \mathrm{~h}$ before the treatment. The adaxial surfaces of the leaves were syringe infiltrated, with approximately $10 \mu \mathrm{l}$ of ClsA $(500 \mu \mathrm{g} / \mathrm{ml})$ purified from the culture supernatant of wild type $X$. oryzae pv. oryzae (strain BXO43; our laboratory wild type) or with buffer (10 $\mathrm{mM}$ potassium phosphate buffer $\mathrm{pH}$ 6.0) alone (as described in Jha et al. (2007)). In this procedure, approximately, 30-40 $\mu \mathrm{l}$ of either ClsA or buffer are taken into a needle less $1 \mathrm{ml}$ syringe for infiltration, and we are able to infiltrate $\sim 10 \mu \mathrm{l}$ of the solution into the leaf while the rest of the solution either falls off the adaxial surface or passes right through to the abaxial surface. Twenty to 30 leaf pieces covering the infiltrated zone from each of the treatments were harvested $12 \mathrm{~h}$ after infiltration. At this time point, HR like symptoms are not observed in ClsA-treated leaves, and it would take a further $24 \mathrm{~h}$ for these symptoms to be visible (Supplementary Fig. S1). The leaf tissues were ground to a fine powder (in liquid nitrogen using a mortar and pestle) and subjected to total RNA isolation by using Trizol (Invitrogen, Carlsbad, CA), following the manufacturer's instructions. The quality of the isolated RNA was assessed by agarose gel electrophoresis and quantitated using a spectrophotometer.

\section{Microarray analysis}

Eight micrograms of total RNA isolated from ClsA- and buffertreated leaves were converted to double-strand cDNA using an Affymetrix (Santa Clara, CA) one cycle labeling kit following the manufacturer's instructions. The synthesized doublestranded cDNA was column purified and was further subjected to RNA amplification using an Affymetrix IVT labeling kit. The amplified RNA (cRNA) generated in this manner was quantitated by using a NanoDrop ${ }^{\circledR}$ ND-1000 UV-Vis Spectrophotometer (NanoDrop Technologies, Wilmington, DE). The normalized cRNA was fragmented (into 50-200 bp fragments), hybridized to rice gene chips, washed, stained, and scanned as per Affymetrix protocols. The experiment was repeated with three different biological replicates using RNA isolated from three batches of rice leaves treated with either $X$. oryzae pv. oryzae ClsA or buffer.

\section{Data analysis}

The data were analyzed by using the GCOS of Affymetrix. The present calls ranged between $40 \%$ and $42 \%$ in ClsAtreated leaves and between $38 \%$ and $40 \%$ in the buffer- treated leaves. All the CEL (cell intensity) files generated by GCOS were uploaded to Avadis ${ }^{\mathrm{TM}}$ software version 4.3 (an Affymetrix approved software manufactured by Strand Life Sciences, Bangalore, India) for further analysis. The CEL files of the buffer-treated samples were grouped as control whereas the ClsA-treated sample files were grouped as treatment. The data were normalized using RMA (Irizarry et al. 2003a, b) and PLIER (Affymetrix Inc 2005) algorithms available in Avadis and subjected to differential expression analysis as per the manufacturer's instructions. The genes identified by both the algorithms as being either $\geq 2.0$ - or $\geq 1.5$-fold differentially expressed with $p<0.05$ were selected. $Q$-value-based significance analysis was performed for rice genes that were commonly differentially expressed at fold change 1.5 and $p<0.05$. $Q$ values were generated from $p$ values for all 862 genes using the online tool (http://genomics.princeton.edu/storeylab/ qvalue/index.html) with settings of interval 0.01 and smoother method with FDR 0.02 (Storey and Tibshirani 2003). GO enrichment analysis was performed to identify overrepresented GO terms amongst 1.5 -fold change differentially expressed rice genes following ClsA treatment. The Affymetrix probe set IDs of the $\geq 1.5$-fold upregulated and downregulated rice genes were subjected to Gene Ontology Enrichment Analysis Software Toolkit analysis using an online tool accessible at http://omicslab.genetics.ac.cn/ GOEAST following default standard setting (Zheng and Wang 2008). The $\geq 2.0$-fold differentially expressed genes were subjected to NCBI BLASTX analysis to manually curate their functions. A gene was assigned the function of its orthologs if it showed significant homology with expect value $\geq 1 \times 10^{-7}$. Based upon the assigned functions, the differentially expressed genes were further categorized into 14 different functional categories as per Bevan et al. (1998) with some minor changes. We did not have any differentially expressed genes that were predicted to be involved in protein destination and storage. Therefore, we dropped this category. Some of the differentially expressed genes were predicted to function in protein synthesis, while others appeared to encode functions involved in protein degradation. We formed a new category called protein synthesis/ turnover into which we clubbed genes involved in protein synthesis as well as protein degradation. Genes related to abiotic stress were put into a new category called "stress" that is distinct from genes related to biotic stress which were placed under the category of "disease/defense." We did this because we wanted to separate genes related to biotic stress from those related to abiotic stress. The genes having unclear, unclassified or no significant similarity in the available databases were grouped together in a category called "others". In order to identify Arabidopsis orthologs of rice genes, BLASTX searches were performed through TAIR (The Arabidopsis Information Resource; www.arabidopsis.org). 
For the $\geq 1.5$-fold differentially expressed genes, the locus information corresponding to the Affymetrix probe set IDs was obtained from the National Science Foundation rice oligonucleotide array project (http://www.ricearray.org/ma trix.search. shtml) through the rice genome server of The Institute for Genome Research, Rockville, MA (Yuan et al. 2005; Ouyang et al. 2007). These were subjected to pathway analysis using RiceCyc 1.2 software (Jaiswal et al. 2006) available at $\mathrm{http}: / / \mathrm{dev}$.gramene.org/pathway.

Data submission

All the six CEL files (generated by GCOS) and the Avadis ${ }^{\mathrm{TM}}$ processed (PLIER and RMA normalized) files are deposited at the NCBI microarray repository, Gene Expression Omnibus (http://www.ncbi.nlm.nih.gov/geo) under the accession number GSE8216 with details provided about the experimental procedures that are minimum information about a microarray experiment compliant.

\section{Real-time PCR}

Two micrograms of freshly isolated RNA were converted into first-strand cDNA as per the recommended protocol by using Superscript ${ }^{\mathrm{TM}}$ II reverse transcriptase (Invitrogen) and oligo-dT primer. One microliter of the 1:100-fold diluted cDNAs were subjected to real-time PCR analysis using SYBR green PCR master mix (Applied Biosystem, Barrington, UK) following the manufacturer's instructions using gene specific primers designed to amplify 130-170 bp fragments of each gene of interest. After $10 \mathrm{~min}$ of initial denaturation at $95^{\circ} \mathrm{C}$, the samples were subjected to the cycling parameters of $95^{\circ} \mathrm{C}$ for $15 \mathrm{~s}, 58^{\circ} \mathrm{C}$ for $30 \mathrm{~s}$, and $72^{\circ} \mathrm{C}$ for $30 \mathrm{~s}$ (for 40 cycles) using a 7900 HT sequence detection system (Applied Biosystems, Foster City, CA). The relative expression of the gene between ClsA and buffer-treated samples was calculated using the $2^{-\mathrm{ddC}} \mathrm{T}$ method with $O s G A P D H$ as an endogenous control (Livak and Schmittgen 2001). The real-time PCR assays were performed at least three times with the same cDNA sample and repeated at least three times with independently isolated RNA samples.

Co-infiltration of ClsA and wild-type $X$. oryzae pv. oryzae into rice leaves

Three milliliters of wild-type $X$. oryzae pv. oryzae cultures were grown to saturation in Peptone Sucrose broth $(\mathrm{pH} 7.2)$ (Ray et al. 2000). The cells were pelleted and washed in double-distilled water following centrifugation. The pelleted cells were resuspended (at a concentration of approximately $3 \times 10^{9}$ cells $/ \mathrm{ml}$ ) in $1 \mathrm{ml}$ of purified $X$. oryzae pv. oryzae ClsA (final conc. $500 \mu \mathrm{g} / \mathrm{ml}$ ) and syringe infiltrated into the leaves as described above. The total RNA isolated from the zone of infiltration in the leaves was subjected to quantitative real-time PCR analysis (as described above). A Student's $t$ test was performed to check the level of significance for this suppression.

\section{Sequence analysis of $O S A P 2 / E R F$}

Gene sequence of OsAP2/ERF (LOC_Os08g36920) was downloaded from TIGR (http://rice.plantbiology.msu.edu/ cgi-bin/gbrowse/rice/), and BLASTX analysis was performed in Rice Genome Annotation Project BLAST Search (http://rice.Plantbiology.msu.edu/blast.shtml). Amino acid sequence of the gene was used to do a domain search (http://www.expasy.ch/prosite). Secondary structure prediction was performed with Jpred web server (http://www. compbio.dundee.ac.uk/www-jpred).

Cloning $O s A P 2 / E R F$ in Agrobacterial T-DNA vector and transfer to Agrobacterium strain LBA4404

OsAP2/ERF full-length gene was PCR amplified by using a pair of primers, each containing a BamHI site and cloned into the BamHI site between CaMV35S promoter and PolyA on pSB11 binary vector (Ramesh et al. 2004) (Supplementary Fig. S3). This construct was transformed into Escherichia coli DH5 $\alpha$ cells. Positive clones were screened using gene and vector-specific primers and confirmed by sequencing. Plasmids from positive clones were further electroporated into electro-competent cells of Agrobacterium LBA4404.

\section{Callose deposition assay}

Rice leaves were syringe infiltrated with one of the following: water, ClsA $(\sim 100 \mu \mathrm{g} / \mathrm{ml}), \mathrm{LBA} 4404 / \mathrm{pSB} 11$, and LBA4404/ pOSAP2/ERF strains (approximately $10^{9} \mathrm{CFU} / \mathrm{ml}$ resuspended in water). After 12 to $15 \mathrm{~h}$, the leaves were heated with lactophenol at $65^{\circ} \mathrm{C}$ to remove chlorophyll, stained with aniline blue for 2 to $3 \mathrm{~h}$, washed with water, mounted on a slide in $50 \%$ glycerol, and analyzed by an Axioplan2 epifluorescence microscope, using a blue filter (excitation wavelength $365 \mathrm{~nm}$ and emission wavelength above $420 \mathrm{~nm}$ long pass [LP]) and $\times 10$ objective (Hauck et al. 2003).

\section{Cell death assay}

Rice seeds were surface sterilized with sodium hypochlorite and germinated under sterile conditions on a filter paper placed on $0.5 \%$ agar in Petri dishes for 3 to 4 days. Root tips, 1 to $2 \mathrm{~cm}$ long, were treated with one of the following: water, ClsA $(\sim 500 \mu \mathrm{g} / \mathrm{ml}), \mathrm{LBA} 4404 / \mathrm{pSB} 11$, and LBA4404/pOsAP2/ERF. Rice roots were treated with LBA4404/pSB11 and LBA4404/ $\mathrm{p} O s A P 2 / E R F$ for $2 \mathrm{~h}$, and roots were subsequently transferred 
to water for another $14 \mathrm{~h}$. After incubation for a total of $16 \mathrm{~h}$ at $28^{\circ} \mathrm{C}$, roots were washed in $1 \mathrm{X}$ PBS and stained with PI by vacuum infiltration for 10 to $15 \mathrm{~min}$. The roots were mounted on a microscopic slide in 50\% glycerol in $1 \mathrm{X}$ PBS; $0.3-\mu \mathrm{m}$ thick longitudinal optical sections were acquired on a Zeiss LSM-510 Meta confocal microscope using 63X oil immersion (NA 1.4) and were further projected to obtain the image of 2 to $3 \mu \mathrm{m}$ total thickness. HeNe laser at $543 \mathrm{~nm}$ excitation and emission above $560 \mathrm{~nm}$ (LP) was used to detect PI internalization. All images were analyzed using LSM software and further edited using Photoshop (Adobe, San Jose, CA, USA).

\section{Rice resistance assay}

The midveins of leaves of approximately 40 days old TN-1 rice plants were injected with 40 to $50 \mu$ l of water alone, LBA4404/ pSB11 or LBA4404/pOsAP2/ERF cells (resuspended in water at $\sim 10^{9}$ cells $/ \mathrm{ml}$ ) using a 1-ml hypodermic syringe and needle. Six hours later, the midveins of the leaves were inoculated with $X$. oryzae pv. oryzae, 2 to $3 \mathrm{~cm}$ below the point of initial injection, by pricking with a needle that had been used to touch a fresh bacterial colony. After 10 days, the leaves were observed for the appearance of visible disease lesions (discoloration of midvein and surrounding regions).

Acknowledgements GJ was supported by a fellowship from the Council of Scientific and Industrial Research. MD was supported by a research associateship from the Department of Biotechnology, Government of India. This work was supported, in part, by a grant to RVS from the Department of Biotechnology. We thank Dr. K. V. Rao for providing us the $\mathrm{pSB} 11$ vector.

\section{References}

Affymetrix Inc. Technical note: Guide to probe logarithmic intensity error (PLIER) estimation. Santa Clara: Affymetrix; 2005.

Ahlfors R, Lang S, Overmyer K, Jaspers P, Brosche M, Tauriainen A, et al. Arabidopsis RADICAL-INDUCED CELL DEATH1 belongs to the WWE protein-protein interaction domain protein family and modulates abscisic acid, ethylene, and methyl jasmonate responses. Plant Cell. 2004;16:1925-37.

Allen MD, Yamasaki K, Ohme-Takagi M, Tateno M, Suzuki M. A novel mode of DNA recognition by a $\beta$-sheet revealed by the solution structure of the GCC-box binding domain in complex with DNA. EMBO J. 1998;17:5484-96.

Altschul SF, Madden TL, Schäffer AA, Zhang J, Zhang Z, Miller W, et al. Gapped BLAST and PSI-BLAST: A new generation of protein database search programs. Nucleic Acids Res. 1997;25:3389-402.

Bent A, Mackey D. Elicitors, effectors, and $R$ genes: The new paradigm and a lifetime supply of questions. Annu Rev Phytopathol. 2007;45:17.1-17.38.

Bessire M, Chassot C, Jacquat AC, Humphry M, Borel S, Petetot JM, et al. A permeable cuticle in Arabidopsis leads to a strong resistance to Botrytis cinerea. EMBO J. 2007;26:2158-68.

Bevan M, Bancroft I, Bent E, Love K, Goodman H, Dean C, et al. Analysis of $1.9 \mathrm{Mb}$ of contiguous sequence from chromosome 4 of Arabidopsis thaliana. Nature. 1998;39:485-8.
Chassot C, Nawrath C, Metraux JP. Cuticular defects lead to full immunity to a major plant pathogen. Plant J. 2007;49:972-80.

Chen Z, Malamy J, Henning J, Conrath U, Sánchez-Casas P, Silva H, et al. Induction, modification, and transduction of the salicylic acid signal in plant defense responses. Proc Natl Acad Sci USA. 1995;92:4134-7.

Chini A, Fonseca S, Fernández G, Adie B, Chico JM, Lorenzo O, et al. The JAZ family of repressors is the missing link in jasmonate signalling. Nature. 2007;448:666-71.

Darvill AG, Albersheim P. Phytoalexins and their elicitors-a defense against microbial infection in plants. Ann Rev Plant Physiol. 1984;35:243-75.

Dean JD, Goodwin PH, Hsiang T. Induction of glutathione $S$ transferase genes of Nicotiana benthamiana following infection by Colletotrichum destructivum and $C$. orbiculare and involvement of one in resistance. J Exp Bot. 2005;56:1525-33.

Desaki Y, Miya A, Venkatesh B, Tsuyumu S, Yamane H, Kaku H, et al. Bacterial lipopolysaccharides induce defense responses associated with programmed cell death in rice cells. Plant Cell Physiol. 2006;47:1530-40.

Falk A, Feys BJ, Frost LN, Jones JDG, Daniels MJ, Parker JE. EDS1, an essential component of $R$ gene-mediated disease resistance in Arabidopsis has homology to eukaryotic lipases. Proc Natl Acad Sci USA. 1999;96:3292-7.

Felix G, Boller T. Molecular sensing of bacteria in plants. The highly conserved RNA-binding motif RNP-1 of bacterial cold shock proteins is recognized as an elicitor signal in tobacco. J Biol Chem. 2003;278:6201-8.

Felix G, Duran JD, Volko S, Boller T. Plants have a sensitive perception system for the most conserved domain of bacterial flagellin. Plant J. 1999;18:265-76.

Gao G, Zhong Y, Guo A, Zhu Q, Tang W, Zheng W, et al. DRTF: A database of rice transcription factors. Bioinformatics. 2006; 22(10):1286-7.

Grant SR, Fisher EJ, Chang JH, Mole BM, Dangl JL. Subterfuge and manipulation: Type III effector proteins of phytopathogenic bacteria. Annu Rev Microbiol. 2006;60:425-49.

Hauck P, Thilmony R, He SY. A Pseudomonas syringe type III effector suppresses cell wall-based extracellular defense in susceptible Arabidopsis plants. Proc Natl Acad Sci USA. 2003;100:8577-82.

He P, Shan L, Sheen J. Elicitation and suppression of microbeassociated molecular pattern-triggered immunity in plant-microbe interactions. Cell Microbiol. 2007;9:1385-96.

Hu J, Qian W, He C. The Xanthomonas oryzae pv. oryzae eglXoB endoglucanase gene is required for virulence to rice. FEMS Microbiol Lett. 2007;269:273-9.

Irizarry RA, Bolstad BM, Collin F, Cope LM, Hobbs B, Speed TP. Summaries of Affymetrix GeneChip probe level data. Nucleic Acids Res. 2003a;31:e15.

Irizarry RA, Hobbs B, Collin F, Beazer-Barclay YD, Antonellis KJ, Scherf U, et al. Exploration, normalization, and summaries of high density oligonucleotide array probe level data. Biostatistics. 2003b;4:249-64.

Jaiswal P, Ni J, Yap I, Ware D, Spooner W, Youens-Clark K, et al. Gramene: A bird's eye view of cereal genomes. Nucleic Acids Res. 2006;34:D717-23.

Jambunathan N, Siani JM, McNellis TW. A humidity-sensitive Arabidopsis Copine mutant exhibits precocious cell death and increased disease resistance. Plant Cell. 2001;13:2225-40.

Jha G, Rajeshwari R, Sonti RV. Bacterial type two secretion system secreted proteins: Double-edged swords for plant pathogens. Mol Plant-Microbe Interact. 2005;18:891-8.

Jha G, Rajeshwari R, Sonti RV. Functional interplay between two Xanthomonas oryzae pv. oryzae secretion systems in modulating virulence on rice. Mol Plant-Microbe Interact. 2007;20:33-40. 
Jirage D, Tootle TL, Reuber TL, Frost LN, Feys BJ, Parker JE, et al. Arabidopsis thaliana PAD4 encodes a lipase-like gene that is important for salicylic acid signaling. Proc Natl Acad Sci USA. 1999;96:13583-8.

Kaku H, Nishizawa Y, Ishii-Minami N, Akimoto-Tomiyama C, Dohmae N, Takio K, et al. Plant cells recognize chitin fragments for defense signaling through a plasma membrane receptor. Proc Natl Acad Sci USA. 2006;103:11086-91.

Keshavarzi M, Soylu S, Brown I, Bonas U, Nicole M, Rossiter J, et al. Basal defenses induced in pepper by lipopolysaccharides are suppressed by Xanthomonas campestris pv. vesicatoria. Mol Plant-Microbe Interact. 2004;17:805-15.

Kiribuchi K, Sugimori M, Takeda M, Otani T, Okada K, Onodera H, et al. RERJ1, a jasmonic acid-responsive gene from rice, encodes a basic helix-loop-helix protein. Biochem Biophys Res Commun. 2004;325:857-63.

Koo YJ, Kim MA, Kim EH, Song JT, Jung C, Moon JK, et al. Overexpression of salicylic acid carboxyl methyltransferase reduces salicylic acid-mediated pathogen resistance in Arabidopsis thaliana. Plant Mol Biol. 2007;64:1-15.

Kumar D, Klessig DF. High-affinity salicylic acid-binding protein 2 is required for plant innate immunity and has salicylic acid-stimulated lipase activity. Proc Natl Acad Sci USA. 2003;100:16101-6.

Kunkel BN, Brooks DM. Cross talk between signalling pathways in plant defense. Curr Opin Plant Biol. 2002;5:325-31.

Kunze G, Zipfel C, Robatzek S, Niehaus K, Boller T, Felix G. The N terminus of bacterial elongation factor Tu elicits innate immunity in Arabidopsis plants. Plant Cell. 2004;16:3496-507.

Larkin RM, Alonso JM, Ecker JR, Chory J. GUN4, a regulator of chlorophyll synthesis and intracellular signaling. Science. 2003;299:902-6.

Lee J, Parthier B, Löbler M. Jasmonate signalling can be uncoupled from abscisic acid signalling in barley: Identification of jasmonate-regulated transcripts which are not induced by abscisic acid. Planta. 1996;199:625-32.

Lee BM, Park YJ, Park DS, Kang HW, Kim JG, Song ES, et al. The genome sequence of Xanthomonas oryzae pathovar oryzae KACC10331, the bacterial blight pathogen of rice. Nucleic Acids Res. 2005;33:577-86.

Lee SW, Han SW, Bartley LE, Ronald PC. Unique characteristics of Xanthomonas oryzae pv. oryzae AvrXa21 and implications for plant innate immunity. Proc Natl Acad Sci USA. 2006;103:18395-400.

Lee SW, Han SW, Sririyanum M, Park CJ, Seo YS, Ronald PC. A type I-secreted, sulfated peptide triggers XA21-mediated innate immunity. Science. 2009;326(5954):850-3.

Li X, Lin H, Zhang W, Zou Y, Zhang J, Tang X, et al. Flagellin induces innate immunity in nonhost interactions that is suppressed by Pseudomonas syringae effectors. Proc Natl Acad Sci USA. 2005;102:12990-5.

Livak KJ, Schmittgen TD. Analysis of relative gene expression data using real- time quantitative PCR and the 2(T) (-Delta Delta C) method. Methods. 2001;25:402-8.

Lorenzo O, Piqueras R, Sánchez-Serrano J, Solano R. ETHYLENE RESPONSE FACTOR1 integrates signals from ethylene and jasmonate pathways in plant defense. Plant Cell. 2003;15:165-78.

Lorenzo O, Chico JM, Sánchez-Serrano JJ, Solano R. JASMONATEINSENSITIVE1 encodes a MYC transcription factor essential to discriminate between different jasmonate-regulated defense responses in Arabidopsis. Plant Cell. 2004;16:1938-50.

Marrs KA. The function and regulation of glutathione $S$-transferases in plants. Annu Rev Plant Physiol Plant Mol Biol. 1996;47:127-58.

Mei C, Qi M, Sheng G, Yang Y. Inducible overexpression of a rice allene oxide synthase gene increases the endogenous jasmonic acid level, $P R$ gene expression, and host resistance to fungal infection. Mol Plant-Microbe Interact. 2006;19:1127-37.
McGrath KC, Dombrecht B, Manners JM, Schenk PM, Edgar CI, Maclean DJ, et al. Repressor- and activator-type ethylene response factors functioning in jasmonate signaling and disease resistance identified via a genome-wide screen of Arabidopsis transcription factor gene expression. Plant Physiol. 2005;139:949-59.

Nakano T, Suzuki K, Fujimura T, Shinshi H. Genome-wide analysis of the ERF gene family in Arabidopsis and rice. Plant Physiol. 2006; 140:411-32.

Nakashima A, Chen L, Thao NP, Fujiwara M, Wong HL, Kuwano M, et al. RACK1 functions in rice innate immunity by interacting with the Rac1 immune complex. The Plant Cell. 2008;20:2265-79.

Newman M-A, Lahaye ER, Parr A, Daniels MJ, Dow JM. Prior exposure to lipopolysaccharide potentiates expression of plant defenses in response to bacteria. Plant J. 2002;29:487-95.

Norman-Setterblad C, Vidal S, Palva ET. Interacting signal pathways control defense gene expression in Arabidopsis in response to cell wall-degrading enzymes from Erwinia carotovora. Mol Plant-Microbe Interact. 2000;13:430-8.

Okamuro JK, Caster B, Villarroel R, Montagu MV, Jofuku KD. The AP2 domain of APETALA2 defines a large new family of DNA binding proteins in Arabidopsis. Proc Natl Acad Sci USA. 1997; 94:7076-81.

Ouyang S, Zhu W, Hamilton J, Lin H, Campbell M, Childs K, et al. The TIGR Rice Genome Annotation Resource: Improvements and new features. Nucleic Acids Res. 2007;35:D883-7.

Palva TK, Holmstrom K-O, Heino P, Palva ET. Induction of plant defense response by exoenzymes of Erwinia carotovora subsp. carotovora. Mol Plant-Microbe Interact. 1993;6:190-6.

Pennell RI, Lamb C. Programmed cell death in plants. Plant Cell. 1997;9:1157-68.

Rajeshwari R, Jha G, Sonti RV. Role of an in planta expressed xylanase of Xanthomonas oryzae pv. oryzae in promoting virulence on rice. Mol Plant Microbe Interact. 2005;18:830-7.

Ramesh S, Nagadhara D, Reddy VD, Rao KV. Production of transgenic indica rice resistant to yellow stem borer and sapsucking insects, using super-binary vectors of Agrobacterium tumefaciens. Plant Sci. 2004;166:1077-85.

Ray SK, Rajeshwari R, Sonti RV. Mutants of Xanthomonas oryzae pv. oryzae deficient in general secretary pathway are virulence deficient and unable to secrete xylanase. Mol Plant-Microbe Interact. 2000;13:394-401.

Ryan CA, Farmer EE. Oligosaccharide signals in plants: A current assessment. Ann Rev Plant Physiol Mol Biol. 1991;42:651-74.

Sakuma Y, Liu Q, Dubouzet JG, Abe H, Shinozaki K, YamaguchiShinozaki K. DNA-binding specificity of the ERF/AP2 domain of Arabidopsis DREBs, transcription factors involved in dehydration- and cold-inducible gene expression. Biochem Biophys Res Commun. 2002;290:998-1009.

Scott ME, Dossani ZY, Sandkvist M. Directed polar secretion of protease from single cells of Vibrio cholerae via the type II secretion pathway. Proc Natl Acad Sci USA. 2001;98:13978-83.

Senf F, Tommassen J, Koster M. Polar secretion of proteins via the Xcp type II secretion system in Pseudomonas aeruginosa. Microbiology. 2008;154:3025-32.

Spoel SH, Koornneef A, Claessens SM, Korzelius JP, Van Pelt JA, Mueller MJ, et al. NPR1 modulates cross-talk between salicylateand jasmonate-dependent defense pathways through a novel function in the cytosol. Plant Cell. 2003;15(3):760-70.

Storey JD, Tibshirani R. Statistical significance for genome-wide experiments. Proc Natl Acad Sci USA. 2003;100:9440-5.

Takahashi H, Kanayama Y, Zheng MS, Kusano M, Hase S, Ikegami M, et al. Antagonistic interactions between the SA and JA signaling pathways in Arabidopsis modulate expression of defense genes and gene-for-gene resistance to cucumber mosaic virus. Plant Cell Physiol. 2004;45(6):803-9. 
Takai R, Isogai A, Takayama S, Che FS. Analysis of flagellin perception mediated by flg22 receptor OsFLS2 in rice. Mol Plant-Microbe Interact. 2008;21(12):1635-42.

Tang D, Simonich MT, Innes RW. Mutations in LACS2, a long-chain acyl- coenzyme A synthetase, enhance susceptibility to avirulent Pseudomonas syringae but confer resistance to Botrytis cinerea in Arabidopsis. Plant Physiol. 2007;144:1093-103.

Thines B, Katsir L, Melotto M, Niu Y, Mandaokar A, Liu G, et al. JAZ repressor proteins are targets of the SCFCOI1 complex during jasmonate signalling. Nature. 2007;448:661-5.

Thomma BPHJ, Eggermont K, Penninckx IAMA, Mauch-Mani B, Vogelsang R, Cammue BPA, et al. Separate jasmonate-dependent and salicylate-dependent defense-response pathways in Arabidopsis are essential for resistance to distinct microbial pathogens. Proc Natl Acad Sci USA. 1998;95:15107-11.

Turner JG, Ellis C, Devoto A. The jasmonate signal pathway. Plant Cell. 2002;14:S153-64.

Vidal S, Eriksson ARB, Montesano M, Denecke J, Palva ET. Cell wall- degrading enzymes from Erwinia carotovora cooperate in the salicylic acid-independent induction of a plant defense response. Mol Plant-Microbe Interact. 1998;11:23-32.

Wang XM, Ma QH. Characterization of a jasmonate-regulated wheat protein related to a beta-glucosidase-aggregating factor. Plant Physiol Biochem. 2005;43:185-92.
Yang B, Sugio A, White FF. $O s 8 N 3$ is a host disease-susceptibility gene for bacterial blight of rice. Proc Natl Acad Sci USA. 2006;103: 10503-8.

Yuan Q, Ouyang S, Wang A, Zhu W, Maiti R, Lin H, et al. The institute for genomic research osal rice genome annotation database. Plant Physiol. 2005;138:18-26.

Zhang H, Huang Z, Xie B, Chen Q, Tian X, Zhang X, et al. The ethylene, jasmonate, abscisic acid- and $\mathrm{NaCl}$-responsive tomato transcription factor JERF1 modulates expression of GCC box-containing genes and salt tolerance in tobacco. Planta. 2004a;220:262-70.

Zhang H, Zhang D, Chen J, Yang Y, Huang Z, Huang D, et al. Tomato stress responsive factor TSRF1 interacts with ethylene-responsive element GCC box and regulates pathogen resistance to Ralstonia solanacearum. Plant Mol Biol. 2004b;55:825-34.

Zheng Q, Wang X. GOEAST: A web-based software toolkit for Gene Ontology enrichment analysis. Nucleic Acids Res. 2008;36: W358-63.

Zipfel C, Robatzek S, Navarro L, Oakeley EJ, Jones JDG, Felix G, et al. Bacterial disease resistance in Arabidopsis through flagellin perception. Nature. 2004;428:764-7.

Zipfel C, Kunze G, Chinchilla D, Caniard A, Jones JDG, Boller T, et al. Perception of the bacterial PAMP EF-Tu by the receptor EFR restricts Agrobacterium-mediated transformation. Cell. 2006; 125:749-60. 